\title{
A New Approach to Adaptive Stabilization of Stochastic High-Order Nonholonomic Systems
}

\author{
Guangju Li $\mathbb{D}^{1}$ and Kemei Zhang $\mathbb{D}^{2}$ \\ ${ }^{1}$ Institute of Automation, Qufu Normal University, Shandong Province 273165, China \\ ${ }^{2}$ School of Mathematics Sciences, Qufu Normal University, Shandong Province 273165, China \\ Correspondence should be addressed to Kemei Zhang; zhkm90@126.com
}

Received 6 May 2018; Accepted 10 July 2018; Published 18 September 2018

Academic Editor: Weihai Zhang

Copyright (C) 2018 Guangju Li and Kemei Zhang. This is an open access article distributed under the Creative Commons Attribution License, which permits unrestricted use, distribution, and reproduction in any medium, provided the original work is properly cited.

\begin{abstract}
This paper studies the problem of adaptive stabilization for a class of stochastic high-order nonholonomic systems. Under the weaker assumptions, by constructing the appropriate Lyapunov function and combining sign function technique, an adaptive state feedback controller is designed to guarantee global asymptotic stability in probability of the closed-loop system. The effectiveness of the controller is demonstrated by a mechanical system.
\end{abstract}

\section{Introduction}

Ever since the stochastic stability theory was established by $[1,2]$, the design and analysis of backstepping controller for stochastic nonlinear systems has achieved remarkable development in recent years; see [1,3-12] and the references therein. But these papers do not consider stochastic nonholonomic systems.

In this paper, we consider stochastic high-order nonholonomic systems

$$
\begin{aligned}
\mathrm{d} x_{0}= & d_{0}(t, \theta) u_{0}^{p_{0}} \mathrm{~d} t+f_{0}\left(x_{0}, \theta\right) \mathrm{d} t+g_{0}^{\top}\left(x_{0}, \theta\right) \mathrm{d} \omega, \\
\mathrm{d} x_{i}= & d_{i}(t, \theta) u_{0}^{q_{i}} x_{i+1}^{p_{i}} \mathrm{~d} t+f_{i}\left(x_{0}, x, \theta\right) \mathrm{d} t \\
& +g_{i}^{\top}\left(x_{0}, x, \theta\right) \mathrm{d} \omega, \\
\mathrm{d} x_{n}= & d_{n}(t, \theta) u_{0}^{q_{n}} u^{p_{n}} \mathrm{~d} t+f_{n}\left(x_{0}, x, \theta\right) \mathrm{d} t \\
& +g_{n}^{\top}\left(x_{0}, x, \theta\right) \mathrm{d} \omega,
\end{aligned}
$$

where $x_{0} \in R$ and $x=\left(x_{1}, \ldots, x_{n}\right)^{\top} \in R^{n}$ are system states, $u_{0}$ and $u$ are control inputs, $p_{i} \in R_{\text {odd }}^{\geq 1} \triangleq$ $\{p / q \mid p$ and $q$ are positive odd integers, and $p \geq q\}$ are odd integers, and $q_{i} \geq 0$ are constants, $i=1, \ldots, n . \theta \in R^{m}$ is an unknown constant vector. $\omega$ is a $r$-dimensional standard Wiener process defined on a probability space $(\Omega, \mathscr{F}, P)$ with
$\Omega$ being a sample space, $\mathscr{F}$ being a filtration, and $P$ being a probability measure. $f_{0}\left(x_{0}, \theta\right): R \times R^{m} \longrightarrow R, g_{0}\left(x_{0}, \theta\right)$ : $R \times R^{m} \longrightarrow R^{r}, f_{i}\left(x_{0}, x, \theta\right): R \times R^{n} \times R^{r} \longrightarrow R, g_{i}\left(x_{0}, x, \theta\right):$ $R \times R^{n} \times R^{r} \longrightarrow R^{r}, i=1, \ldots, n$, are locally Lipschitz functions. $d_{i}(t, \theta), i=0, \ldots, n$, are nonlinear control coefficients.

Since many mechanical systems can be modeled by system (1), a series of theoretical results have been obtained. To mention a few, [13-16] investigated the deterministic case of $g_{i}=0$. Specially when $d_{i}=p_{i}=q_{i}=1$ and $f_{i}=0$, the authors designed a continuous controller by proposing a sliding mode control approach in [13]. When systems contain nonlinear drifts and unknown time-varying coefficients, [15] presented an adaptive control approach to achieve sate-feedback stabilization. High-order nonholonomic systems, that is, $p_{i} \geq$ 1 , were introduced in [16]. The design procedure in [16] combined the idea of a discontinuous change of coordinate and adding a power integrator. However, it did not consider nonlinear parameterizations. Because many practical control systems such as biochemical processes and machines with friction often contain unknown parameters, [14] studied the problem of adaptive stabilization control design for a class of high-order nonholonomic systems with strong nonlinear drifts. Since stochastic noise frequently arises and is inevitable in practical control systems, how to extend these approaches to stochastic high-order nonholonomic systems is a very interesting problem. When $g_{i} \neq 0$, [17] studied the problem of 
state feedback stabilization for a class of high-order stochastic nonholonomic systems with disturbed control directions and more general nonlinear drifts. However, nonlinear parameterization and nonlinear drift term in $x_{0}$-subsystem were not discussed in [17]. Unfortunately, all these mentioned results require that the nonlinearities $f_{i}(\cdot)$ are dependent on $\left(x_{1}, \ldots, x_{i}\right)$. Naturally, an interesting problem is put forward: For system (1), under weaker assumptions, can a stabilizable state feedback controller be designed?

In this paper, we will provide a satisfactory answer to this problem. By constructing the appropriate Lyapunov function, skillfully combining parameter separation, sign function, and backstepping design approach, an adaptive state feedback controller is designed to guarantee global asymptotic stability in probability of the closed-loop system. Finally, a simulation example is used to demonstrate the effectiveness of this approach.

The contributions and difficulties of this paper are highlighted from three aspects.

(i) The system under consideration is more general than those investigated in [13-17]. System (1) has unknown control coefficients and permits unknown parameters to enter nonlinear equations. Nonlinear functions $f_{i}(\cdot)$ are dependent on $\left(x_{1}, \ldots, x_{i+1}\right)$, which makes discontinuous change of coordinates be inapplicable to the adaptive state feedback control of systems (1). In this paper, we propose a novel design approach to solve this obstacle.

(ii) The unknown growth rates of the upper bounds of $f_{i}(\cdot)$ and $g_{i}(\cdot)$ are extended; see Remark 13. Therefore, some new mathematical tools, such as sign function and transformation technique, are introduced to simplify the construction of Lyapunov function.

(iii) An practical example for mobile robot with small angle measurement error is modeled and solved by the proposed approach.

This paper is organized as follows. Section 2 gives some preliminaries. Section 3 presents the design and analysis of the adaptive controller, following a practical example in Section 4. Section 5 concludes this paper. The proofs of Propositions 16-19 are given in Appendix.

\section{Mathematical Preliminaries}

Some notations and definitions will be used throughout this paper. $R^{+}$stands for the set of all nonnegative real numbers and $R^{n}$ denotes real $n$-dimensional space. For vector $\chi=$ $\left(\chi_{1}, \ldots, \chi_{n}\right)^{\top}, \bar{\chi}_{i}$ represents $\left(\chi_{1}, \ldots, \chi_{i}\right)^{\top}$. For a given vector or matrix $x, x^{\top}$ denotes its transpose, $|x|$ is the Euclidean norm of a vector $x$, and $\operatorname{Tr}\{x\}$ denotes its trace when $x$ is square. $\mathscr{C}^{i}$ denotes the set of all functions with continuous $i$ th partial derivatives. $\mathscr{K}$ denotes the set of all functions: $R^{+} \longrightarrow R^{+}$ that are continuous, strictly increasing, and vanishing at zero; $\mathscr{K}_{\infty}$ denotes the set of all functions that are of class $\mathscr{K}$ and unbounded. For any $y \in R$, sign function $\operatorname{sgn}(y)$ is defined as $\operatorname{sgn}(y)=1$ if $y>0 ; \operatorname{sgn}(y)=0$ if $y=0$ and $\operatorname{sgn}(y)=-1$ if $y<0$. For simplicity, we denote $\left\lceil\left. y\right|^{p}=|y|^{p} \operatorname{sgn}(y)\right.$. The arguments of functions are sometimes omitted or simplified, for example, a function $f(x(t))$ is denoted by $f(x)$ or $f$.
In what follows, we present some definitions and lemmas which will be frequently used in the design and analysis of controller. Consider stochastic nonlinear system

$$
\begin{aligned}
\mathrm{d} x & =f(x, \theta) \mathrm{d} t+g(x, \theta) \mathrm{d} \omega, \\
x\left(t_{0}\right) & =x_{0} \in R^{n},
\end{aligned}
$$

where $\omega$ is a $r$-dimensional standard Wiener process defined on a probability space $(\Omega, \mathscr{F}, P), x \in R^{n}$ is system state, $\theta \in$ $R^{m}$ is an unknown constant vector and the functions $f: R^{n} \times$ $R^{m} \longrightarrow R^{n}$, and $g: R^{n} \times R^{m} \longrightarrow R^{n \times r}$ are locally bounded and locally Lipschitz with respect to $x$.

Definition 1 (see [1]). The equilibrium $x(t)=0$ of system (2) with $f(0, \theta)=0, g(0, \theta)=0$ is

(i) globally stable in probability if for any $\varepsilon>0$, there exists a class $\mathscr{K}$ function $r(\cdot)$ such that $P\left\{|x(t)|<r\left(x_{0}\right)\right\} \geq$ $1-\varepsilon$, for any $t \geq 0, x_{0} \in R^{n} \backslash\{0\}$

(ii) globally asymptotically stable in probability if it is globally stable in probability and $P\left\{\lim _{t \rightarrow \infty}|x(t)=0|\right\}=1$, for any $x_{0} \in R^{n}$

Lemma 2 (see [1]). For system (2), if there exists a $\mathscr{C}^{2}$ function $V(x): R^{n} \longrightarrow R^{+}$and class $\mathscr{K}_{\infty}$ functions $\alpha_{1}(\cdot)$ and $\alpha_{2}(\cdot)$, such that

$$
\begin{aligned}
\alpha_{1}(|x|) \leq & V(x) \leq \alpha_{2}(|x|) \\
\mathscr{L} V(x)= & \frac{\partial V(x)}{\partial x} f(x, \theta) \\
& +\frac{1}{2} \operatorname{Tr}\left\{g^{T}(x, \theta) \frac{\partial^{2} V(x)}{\partial x^{2}} g(x, \theta)\right\} \\
\leq & -W(x),
\end{aligned}
$$

where $W(x)$ is a nonnegative continuous function, then, for any $x\left(t_{0}\right) \in R^{n}$,

(i) there exists an almost surely unique solution on $\left[t_{0}, \infty\right)$

(ii) the equilibrium $x=0$ is globally stable in probability, and the solution $x(t)$ satisfies $P\left\{\lim _{t \rightarrow \infty} W(x(t))=0\right\}=1$

Lemma 3 (see [18]). For any real-valued continuous function $h(x, y)$, where $x \in R^{m}, y \in R^{n}$, there are smooth scalar functions $c(x) \geq 1$ and $d(y) \geq 1$, such that $|h(x, y)| \leq$ $c(x) d(y)$.

Lemma 4 (see [19]). Let $x, y$ be real variables, then for any positive real numbers $b, c, d$ and continuous function $a(\cdot) \geq 0$, one has $a(\cdot)|x|^{c}|y|^{d} \leq b|x|^{c+d}+(d /(c+d))((c+$ d)/c) $)^{-c / d} a(\cdot)^{(c+d) / d} b^{-c / d}|y|^{c+d}$.

Lemma 5 (see [20]). $f(x)=\lceil x\rceil^{a}$ is continuously differentiable and $\dot{f}(x)=a|x|^{a-1}$, where $a \geq 1, x \in R$. Moreover, $d f(x(t)) / d t=a|x(t)|^{a-1} \dot{x}(t)$.

Lemma 6 (see [20]). If $p=a / b \in R_{\text {odd }}^{\geq 1}, b \geq 1$, then $\left|x^{p}-y^{p}\right| \leq$ $2^{1-1 / b}\left|\lceil x\rceil^{a}-\lceil y\rceil^{a}\right|^{1 / b}$ for any $x, y \in R$.

Lemma 7 (see [20]). For given $r \geq 0$ and every $x \in R, y \in R$, $|x+y|^{r} \leq C_{r}\left(|x|^{r}+|y|^{r}\right)$ holds, where $C_{r}=2^{r-1}$ if $r \geq 1$ and $C_{r}=1$ if $0 \leq r<1$. 
Lemma 8 (see [20]). Let $f:[a, b] \longrightarrow R$ be a continuous monotone function with $f(a)=0$; then $\left|\int_{a}^{b} f(x) d x\right| \leq$ $|f(b)||b-a|$.

\section{Design and Analysis of Adaptive Controller}

3.1. Problem Formulation and Assumptions. The aim of this paper is to design an adaptive state feedback controller for system (1) to guarantee global asymptotic stability in probability of the closed-loop system. We need the following assumptions to achieve this aim.

Assumption 9. The sign of $d_{i}(t, \theta), i=0, \ldots, n$, is assumed to be positive, and there exist smooth functions $0<\lambda_{i, 1}(t) \leq$ $\lambda_{i, 2}(t, \theta)$, such that

$$
0<\lambda_{i, 1}(t) \leq d_{i}(t, \theta) \leq \lambda_{i, 2}(t, \theta) .
$$

Assumption 10. For smooth functions $f_{0}\left(x_{0}, \theta\right)$ and $g_{0}\left(x_{0}, \theta\right)$, there exist bounded smooth functions $\alpha_{0}\left(x_{0}, \theta\right), \beta_{0}\left(x_{0}, \theta\right)$ such that

$$
\begin{aligned}
& f_{0}\left(x_{0}, \theta\right)=x_{0} \alpha_{0}\left(x_{0}, \theta\right), \\
& g_{0}\left(x_{0}, \theta\right)=x_{0} \beta_{0}\left(x_{0}, \theta\right) .
\end{aligned}
$$

Assumption 11. For $i=1, \ldots, n$, there exist nonnegative continuous functions $\alpha_{i}\left(x_{0}, \bar{x}_{i}, \theta\right), \beta_{i}\left(x_{0}, \bar{x}_{i}, \theta\right)$ and a constant $\tau$ satisfying $-r_{n}<\tau<0$ such that

$$
\begin{aligned}
& \left|f_{i}\left(x_{0}, x, \theta\right)\right| \\
& \quad \leq\left(\left|x_{1}\right|^{\left(r_{i}+\tau\right) / r_{1}}+\cdots+\left|x_{i}\right|^{\left(r_{i}+\tau\right) / r_{i}}\right) \alpha_{i}\left(x_{0}, \bar{x}_{i}, \theta\right) \\
& \quad+c_{i} u_{0}^{q_{i}} d_{i}(t, \theta)\left|x_{i+1}\right|^{\left(r_{i}+\tau\right) / r_{i+1}}, \\
& \left|g_{i}\left(x_{0}, x, \theta\right)\right| \\
& \quad \leq\left(\left|x_{1}\right|^{\left(2 r_{i}+\tau\right) / 2 r_{1}}+\cdots+\left|x_{i}\right|^{\left(2 r_{i}+\tau\right) / 2 r_{i}}\right) \beta_{i}\left(x_{0}, \bar{x}_{i}, \theta\right),
\end{aligned}
$$

where $r_{n+1}$ is a given positive constant and $r_{1}, \cdots, r_{n}$ are recursively defined by $r_{i}=p_{i} r_{i+1}-\tau$ and nonnegative constants $c_{i}<1 / 2^{p_{i}-1}$.

Remark 12. Assumptions 10-11 imply that $f_{0}(0, \theta)=0$, $g_{0}(0, \theta)=0, f_{i}(0,0, \theta)=0, g_{i}(0,0, \theta)=0, i=1, \ldots, n$; that is, origin is the equilibrium of system (1).

Remark 13. Assumption 11 enlarges the scope of nonholonomic systems. Specifically, if state $x_{i+1}$ does not appear in the nonlinear function $f_{i}(\cdot)$, Assumption 11 is degenerated into the following form:

$$
\begin{aligned}
& \left|f_{i}\left(x_{0}, x, \theta\right)\right| \\
& \quad \leq\left(\left|x_{1}\right|^{\left(r_{i}+\tau\right) / r_{1}}+\cdots+\left|x_{i}\right|^{\left(r_{i}+\tau\right) / r_{i}}\right) \alpha_{i}\left(x_{0}, \bar{x}_{i}, \theta\right), \\
& \left|g_{i}\left(x_{0}, x, \theta\right)\right| \\
& \quad \leq\left(\left|x_{1}\right|^{\left(2 r_{i}+\tau\right) / 2 r_{1}}+\cdots+\left|x_{i}\right|^{\left(2 r_{i}+\tau\right) / 2 r_{i}}\right) \beta_{i}\left(x_{0}, \bar{x}_{i}, \theta\right),
\end{aligned}
$$

where $r_{1}=1, r_{i}=1+(i-1) \tau$ with $\tau$ being a constant for each $i=1, \ldots, n$. Particularly, when $\tau=0$, (7) becomes the following growth condition:

$$
\begin{aligned}
& \left|f_{i}\left(x_{0}, x, \theta\right)\right| \leq\left(\left|x_{1}\right|+\cdots+\left|x_{i}\right|\right) \alpha_{i}\left(x_{0}, \bar{x}_{i}, \theta\right), \\
& \left|g_{i}\left(x_{0}, x, \theta\right)\right| \leq\left(\left|x_{1}\right|+\cdots+\left|x_{i}\right|\right) \beta_{i}\left(x_{0}, \bar{x}_{i}, \theta\right) .
\end{aligned}
$$

If $\alpha_{i}\left(x_{0}, \bar{x}_{i}, \theta\right)$ and $\beta_{i}\left(x_{0}, \bar{x}_{i}, \theta\right)$ are constants, (8) becomes the linear growth condition in [21]. If $\alpha_{i}\left(x_{0}, \bar{x}_{i}, \theta\right)$ and $\beta_{i}\left(x_{0}, \bar{x}_{i}, \theta\right)$ are smooth nonnegative functions, (8) becomes the linear growth condition in $[22,23]$. When $\tau=-p / q \in(-2 /(4 n+$ $1), 0)$ with $p$ being an even integer and $q$ being an odd integer, condition (7) becomes the low-order growth condition in $[24,25]$. Assumption 11 extends the value of $\tau$ to an explicit interval rather than a ratio of an even integer over an odd integer.

Remark 14. By Lemma 3, there are smooth scalar functions $\tilde{\lambda}_{i, 2}(t) \geq 1, \bar{\alpha}_{i}\left(x_{0}, \bar{x}_{i}\right) \geq 1, \bar{\beta}_{i}\left(x_{0}, \bar{x}_{i}\right) \geq 1, i=0, \ldots, n$, and $\Theta(\theta) \geq 1$ such that

$$
\begin{aligned}
& \lambda_{i, 1}(t) \leq d_{i}(t, \theta) \leq \tilde{\lambda}_{i, 2}(t) \Theta, \\
& \left|f_{0}\left(x_{0}, \theta\right)\right| \leq\left|x_{0}\right| \bar{\alpha}_{0}\left(x_{0}\right) \Theta, \\
& \left|g_{0}\left(x_{0}, \theta\right)\right| \leq\left|x_{0}\right| \bar{\beta}_{0}\left(x_{0}\right) \Theta, \\
& \left|f_{i}\left(x_{0}, x, \theta\right)\right| \\
& \quad \leq\left(\left|x_{1}\right|^{\left(r_{i}+\tau\right) / r_{1}}+\cdots+\left|x_{i}\right|^{\left(r_{i}+\tau\right) / r_{i}}\right) \bar{\alpha}_{i}\left(x_{0}, \bar{x}_{i}\right) \Theta \\
& \quad+c_{i} u_{0}^{q_{i}} d_{i}(t, \theta)\left|x_{i+1}\right|^{\left(r_{i}+\tau\right) / r_{i+1}}, \\
& \quad\left|g_{i}\left(x_{0}, x, \theta\right)\right| \\
& \quad \leq\left(\left|x_{1}\right|^{\left(2 r_{i}+\tau\right) / 2 r_{1}}+\cdots+\left|x_{i}\right|^{\left(2 r_{i}+\tau\right) / 2 r_{i}}\right) \bar{\beta}_{i}\left(x_{0}, \bar{x}_{i}\right) \Theta .
\end{aligned}
$$

3.2. Stability and Convergence Analysis. We state the main result in this paper.

Theorem 15. If Assumptions 9-11 hold for system (1), under an appropriate controller, the origin of the closed-loop system is globally asymptotically stable in probability for any initial condition.

Proof. The proof is based on inductive argument. Firstly, we design an adaptive controller by considering two cases: $x_{0}\left(t_{0}\right) \neq 0$ and $x_{0}\left(t_{0}\right)=0$.

Case I $\left(x_{0}\left(t_{0}\right) \neq 0\right)$. The structure of system (1) means that the design procedure is divided into two separate parts.

Part I (design of controller $u_{0}$ ). Let us consider $x_{0}$-subsystem in system (1), define $\Theta_{0}=\lambda_{0,1}^{-1} \max \left\{\lambda_{0,1}^{-1}, \Theta, \Theta^{2}\right\}$, and choose $V_{0}=x_{0}^{4} / 4+\left(\lambda_{0,1} / 2\right) \widetilde{\Theta}_{0}^{2}$, where $\widetilde{\Theta}_{0}=\Theta_{0}-\widehat{\Theta}_{0}$. From Remark 14, it follows that

$$
\begin{aligned}
\mathscr{L} V_{0} & =x_{0}^{3}\left(d_{0} u_{0}^{p_{0}}+f_{0}\right)+\frac{3}{2} \operatorname{Tr}\left\{g_{0} x_{0}^{2} g_{0}^{\top}\right\}-\lambda_{0,1} \widetilde{\Theta}_{0} \dot{\widehat{\Theta}}_{0} \\
& \leq-\frac{a}{\lambda_{0,1}} x_{0}^{4}+\frac{a}{\lambda_{0,1}} x_{0}^{4}+x_{0}^{3} d_{0} u_{0}^{p_{0}}+\Theta \bar{\alpha}_{0}\left(x_{0}\right) x_{0}^{4}
\end{aligned}
$$




$$
\begin{aligned}
& +\frac{3}{2} \Theta^{2} \bar{\beta}_{0}^{2}\left(x_{0}\right) x_{0}^{4}-\lambda_{0,1} \widetilde{\Theta}_{0} \dot{\widehat{\Theta}}_{0} \\
\leq & -\frac{a}{\lambda_{0,1}} x_{0}^{4}+\lambda_{0,1} x_{0}^{3}\left(\frac{d_{0}}{\lambda_{0,1}} u_{0}^{p_{0}}+h\left(x_{0}\right) \widehat{\Theta}_{0} x_{0}\right) \\
& -\lambda_{0,1} \widetilde{\Theta}_{0}\left(\dot{\widehat{\Theta}}_{0}-x_{0}^{4} h\left(x_{0}\right)\right),
\end{aligned}
$$

where $a$ is a positive constant and $h\left(x_{0}\right)=a+\bar{\alpha}_{0}\left(x_{0}\right)+$ $(3 / 2) \bar{\beta}_{0}^{2}\left(x_{0}\right)$ is a positive smooth function. Substituting the adaptive controller

$$
\begin{aligned}
u_{0} & =-\left(x_{0} h\left(x_{0}\right) \sqrt{\widehat{\Theta}_{0}^{2}+1}\right)^{1 / p_{0}}, \\
\dot{\widehat{\Theta}}_{0} & =x_{0}^{4} h\left(x_{0}\right)
\end{aligned}
$$

into (10) leads to

$$
\mathscr{L} V_{0} \leq-\frac{a}{\lambda_{0,1}} x_{0}^{4}
$$

The succeeding proposition characterizes the features of $x_{0}$ subsystem.

Proposition 16. If Assumptions 9-10 hold for $x_{0}$-subsystem, controller (11) guarantees that

(i) $x_{0}$-subsystem has an almost surely unique solution on $\left[t_{0}, \infty\right)$ for any $x_{0}\left(t_{0}\right) \neq 0$

(ii) the equilibrium $x_{0}=0$ of $x_{0}$-subsystem is globally asymptotically stable in probability

(iii) the solution of $x_{0}$-subsystem and $u_{0}$ does not cross zero for any $x_{0}\left(t_{0}\right) \neq 0$

Proof. See Appendix.

Part II (design of controller $u$ ). In this part, we need to consider $x_{i}$-subsystem in system (1). With the help of Proposition 16, controller $u$ will be recursively constructed by applying the adding a power integrator approach. Before the beginning of recursive design, we define $\Theta_{1}=\max \left\{1, \Theta, \Theta^{2}\right\}$ and state transformation

$$
\begin{gathered}
\xi_{1}=\left\lceil x_{1}\right\rceil^{\sigma / r_{1}}-\left\lceil x_{1}^{*}\right\rceil^{\sigma / r_{1}}, \quad x_{1}^{*}=0, \\
\xi_{2}=\left\lceil x_{2}\right\rceil^{\sigma / r_{2}}-\left\lceil x_{2}^{*}\right\rceil^{\sigma / r_{2}}, \\
x_{2}^{*}=-\phi_{1}\left(x_{0}, x_{1}, \widehat{\Theta}_{1}\right)\left\lceil\xi_{1}\right\rceil^{r_{2} / \sigma}, \\
\vdots \\
\xi_{k}=\left\lceil x_{k}\right\rceil^{\sigma / r_{k}}-\left\lceil x_{k}^{*}\right\rceil^{\sigma / r_{k}}, \\
x_{k}^{*}=-\phi_{k-1}\left(x_{0}, \bar{x}_{k-1}, \widehat{\Theta}_{1}\right)\left\lceil\xi_{k-1}\right\rceil^{r_{k} / \sigma},
\end{gathered}
$$

where $\sigma$ is a positive constant satisfying the relationship $\sigma \geq$ $\max \left\{p_{1} r_{2}, \ldots, p_{n} r_{n+1}\right\}, \widehat{\Theta}_{1}$ is the estimate of $\Theta_{1}$, and functions $\phi_{k}(\cdot)$ satisfying $\operatorname{sgn}\left(\phi_{k}\right)=\operatorname{sgn}\left(u_{0}^{q_{k}}\right)$ will be determined later.
To solve the problem caused by sign function, the definition of $W_{k}\left(x_{0}, \bar{x}_{k}, \widehat{\Theta}_{1}\right)$ is given by

$$
\begin{aligned}
W_{k} & \left(x_{0}, \bar{x}_{k}, \widehat{\Theta}_{1}\right) \\
= & \int_{x_{k}^{*}}^{x_{k}}\left\lceil\lceil s\rceil^{\sigma / r_{k}}-\left\lceil x_{k}^{*}\right\rceil^{\sigma / r_{k}}\right\rceil^{\left(4 l \sigma-\tau-r_{k}\right) / \sigma} \mathrm{d} s,
\end{aligned}
$$

where $k=1, \ldots, n$ and $4 l$ is an even number and satisfies $(4 l-2) \sigma \geq \tau+r_{k}$.

Proposition 17. $W_{k}$ is $\mathscr{C}^{2}$ function and satisfies $C_{k 1} \mid x_{k}-$ $\left.x_{k}^{*}\right|^{(4 l \sigma-\tau) / r_{k}} \leq W_{k} \leq C_{k 2}\left|\xi_{k}\right|^{(4 l \sigma-\tau) / \sigma}$, where $C_{k 1}=$ $2^{\left(\sigma-r_{k}\right)\left(4 l \sigma-\tau-r_{k}\right) / \sigma r_{k}}\left(r_{k} /(4 l \sigma-\tau)\right), C_{k 2}=2^{1-r_{k} / \sigma}, k=1, \ldots, n$.

Proof. See Appendix.

To obtain the expression of $u$, we determine $\phi_{1}, \cdots, \phi_{n}$ by induction, the design procedure is implemented as follows.

Step 1. Let $V_{1}=W_{1}+(1 / 2) \widetilde{\Theta}_{1}^{2}$, where $\widetilde{\Theta}_{1}=\Theta_{1}-\widehat{\Theta}_{1}$. By using (13), (A.3), (A.7), and Lemma 7, we get

$$
\begin{aligned}
& \mathscr{L} V_{1} \leq\left\lceil\xi_{1}\right\rceil^{\left(4 l \sigma-\tau-r_{1}\right) / \sigma}\left(d_{1} u_{0}^{q_{1}} x_{2}^{p_{1}}+f_{1}\right) \\
& +\frac{4 l \sigma-\tau-r_{1}}{2 r_{1}}\left|\xi_{1}\right|^{\left((4 l-1) \sigma-\tau-r_{1}\right) / \sigma}\left|x_{1}\right|^{\left(\sigma-r_{1}\right) / r_{1}}\left|g_{1}\right|^{2} \\
& \quad-\widetilde{\Theta}_{1} \dot{\widehat{\Theta}}_{1} \leq-n \xi_{1}^{4 l}+n \xi_{1}^{4 l} \\
& +\left\lceil\xi_{1}\right\rceil^{\left(4 l \sigma-\tau-r_{1}\right) / \sigma} d_{1} u_{0}^{q_{1}}\left(x_{2}^{p_{1}}-x_{2}^{* p_{1}}\right) \\
& +\left\lceil\xi_{1}\right\rceil^{\left(4 l \sigma-\tau-r_{1}\right) / \sigma} d_{1} u_{0}^{q_{1}} x_{2}^{* p_{1}}-\widetilde{\Theta}_{1} \dot{\widehat{\Theta}}_{1} \\
& +\left.\frac{4 l \sigma-\tau-r_{1}}{2 \xi_{1}}\left|\xi_{1}^{\left.(4 l-1) \sigma-\tau-r_{1}\right) / \sigma}\right| x_{1}\right|^{\left(\sigma+r_{1}+\tau\right) / r_{1}} \bar{\beta}_{1}^{2} \Theta^{2} \\
& +\left\lceil\xi_{1}\right\rceil^{\left(4 l \sigma-\tau-r_{1}\right) / \sigma} \\
& +\left(\left|x_{1}\right|^{\left(r_{1}+\tau\right) / r_{1}} \bar{\alpha}_{1} \Theta+c_{1} d_{1} u_{0}^{q_{1}}\left|x_{2}\right|^{p_{1}}\right) .
\end{aligned}
$$

Clearly, the application of the fact $\left\lceil\xi_{1}\right\rceil^{\left(4 l \sigma-\tau-r_{1}\right) / \sigma} d_{1} u_{0}^{q_{1}} x_{2}^{* p_{1}} \leq$ 0 implies

$$
\begin{aligned}
& \left\lceil\left.\xi_{1}\right|^{\left(4 l \sigma-\tau-r_{1}\right) / \sigma} u_{0}^{q_{1}} d_{1} x_{2}^{* p_{1}}\right. \\
& \quad+\left\lceil\left.\xi_{1}\right|^{\left(4 l \sigma-\tau-r_{1}\right) / \sigma} c_{1} d_{1} u_{0}^{q_{1}}\left|x_{2}\right|^{p_{1}}\right. \\
& \left.\quad \leq \mid \xi_{1}\right\rceil^{\left(4 l \sigma-\tau-r_{1}\right) / \sigma} u_{0}^{q_{1}} d_{1} x_{2}^{* p_{1}} \\
& \quad+\left|\xi_{1}\right|^{\left(4 l \sigma-\tau-r_{1}\right) / \sigma} \bar{c}_{1} d_{1}\left|u_{0}\right|^{q_{1}}\left|x_{2}^{*}\right|^{p_{1}} \\
& \quad+\left|\xi_{1}\right|^{\left(4 l \sigma-\tau-r_{1}\right) / \sigma} \bar{c}_{1} d_{1}\left|u_{0}\right|^{q_{1}}\left|\xi_{2}\right|^{\left(r_{1}+\tau\right) / \sigma} \\
& \left.\quad \leq \mid \xi_{1}\right\rceil^{\left(4 l \sigma-\tau-r_{1}\right) / \sigma} d_{1} u_{0}^{q_{1}}\left(1-\bar{c}_{1}\right) x_{2}^{* p_{1}} \\
& \quad+\left|\xi_{1}\right|^{\left(4 l \sigma-\tau-r_{1}\right) / \sigma} \bar{c}_{1} d_{1}\left|u_{0}\right|^{q_{1}}\left|\xi_{2}\right|^{\left(r_{1}+\tau\right) / \sigma},
\end{aligned}
$$


where $\bar{c}_{1}=2^{p_{1}-1} c_{1}$ is a positive constant. Substituting (16) into (15) and choosing

$$
\begin{aligned}
x_{2}^{*} & =-\left(\frac{1}{\lambda_{1,1} u_{0}^{q_{1}}\left(1-\bar{c}_{1}\right)}\right. \\
& \left.. \widehat{\Theta}_{1}\left(\bar{\alpha}_{1}+n+\frac{4 l \sigma-\tau-r_{1}}{2 r_{1}} \bar{\beta}_{1}^{2}\right)\right)^{1 / p_{1}}\left\lceil\xi_{1}\right\rceil^{r_{2} / \sigma} \\
& \triangleq-\phi_{1}\left(x_{0}, x_{1}, \widehat{\Theta}_{1}\right)\left\lceil\xi_{1}\right\rceil^{r_{2} / \sigma},
\end{aligned}
$$

one has

$$
\begin{aligned}
\mathscr{L} V_{1} \leq & -n \xi_{1}^{4 l}+\left\lceil\xi_{1}\right]^{\left(4 l \sigma-\tau-r_{1}\right) / \sigma} d_{1} u_{0}^{q_{1}}\left(x_{2}^{p_{1}}-x_{2}^{* p_{1}}\right) \\
& +\left(\Psi_{1}-\dot{\widehat{\Theta}}_{1}\right)\left(\widetilde{\Theta}_{1}+\eta_{1}\right) \\
& +\bar{c}_{1} d_{1}\left|u_{0}\right|^{q_{1}}\left|\xi_{1}\right|^{\left(4 l \sigma-\tau-r_{1}\right) / \sigma}\left|\xi_{2}\right|^{\left(r_{1}+\tau\right) / \sigma}
\end{aligned}
$$

where $\Psi_{1}=\xi_{1}^{4 l}\left(\bar{\alpha}_{1}+n+\left(\left(4 l \sigma-\tau-r_{1}\right) / 2 r_{1}\right) \bar{\beta}_{1}^{2}\right) \geq 0$ and $\eta_{1}=0$.

Step $k(k=2, \ldots, n)$. At Step $k-1$, assume that there exists a $\mathscr{C}^{2}$ function $V_{k-1}$ and virtual controllers $x_{2}^{*}, \cdots, x_{k}^{*}$ such that

$$
\begin{aligned}
\mathscr{L} V_{k-1} & \\
\leq & -(n-k+2) \sum_{i=1}^{k-1} \xi_{i}^{4 l} \\
& +\left[\xi_{k-1}\right]^{\left(4 l \sigma-\tau-r_{k-1}\right) / \sigma} d_{k-1} u_{0}^{q_{k-1}}\left(x_{k}^{p_{k-1}}-x_{k}^{* p_{k-1}}\right) \\
& +\left(\Psi_{k-1}-\dot{\widehat{\Theta}}_{1}\right)\left(\widetilde{\Theta}_{1}+\eta_{k-1}\right) \\
& +\bar{c}_{k-1} d_{k-1}\left|u_{0}\right|^{q_{k-1}}\left|\xi_{k-1}\right|^{\left(4 l \sigma-\tau-r_{k-1}\right) / \sigma}\left|\xi_{k}\right|^{\left(r_{k-1}+\tau\right) / \sigma},
\end{aligned}
$$

where $\bar{c}_{k-1}=2^{p_{k-1}-1} c_{k-1}$ is a nonnegative constant and

$$
0 \leq \Psi_{k-1} \leq\left(\xi_{1}^{4 l}+\cdots+\xi_{k-1}^{4 l}\right) \varphi_{k-1},
$$

for a $\mathscr{C}^{\infty}$ function $\varphi_{k-1} \geq 0$. In what follows, we prove that (19) also holds at Step $k$. To prove this point, considering $V_{k}=$ $V_{k-1}+W_{k}$, we deduce from (19) and (A.3)-(A.13) that

$$
\begin{aligned}
& \mathscr{L} V_{k} \\
& \leq- \\
& \quad+n-k+2) \sum_{i=1}^{k-1} \xi_{i}^{4 l} \\
& +\quad\left[\left.\xi_{k-1}\right|^{\left(4 l \sigma-\tau-r_{k-1}\right) / \sigma} d_{k-1} u_{0}^{q_{k-1}}\left(x_{k}^{p_{k-1}}-x_{k}^{* p_{k-1}}\right)\right. \\
& \quad+\frac{\partial W_{k}}{\partial \widehat{\Theta}_{1}} \dot{\Theta}_{1}+\left(\Psi_{k-1}-\dot{\widehat{\Theta}}_{1}\right)\left(\widetilde{\Theta}_{1}+\eta_{k-1}\right) \\
& +\bar{c}_{k-1} d_{k-1}\left|u_{0}\right|^{q_{k-1}}\left|\xi_{k-1}\right|^{\left(4 l \sigma-\tau-r_{k-1}\right) / \sigma}\left|\xi_{k}\right|^{\left(r_{k-1}+\tau\right) / \sigma} \\
& +\frac{\partial W_{k}}{\partial x_{0}}\left(d_{0} u_{0}^{p_{0}}+f_{0}\right)+\frac{\partial W_{k}}{\partial x_{k}}\left(d_{k} u_{0}^{q_{k}} x_{k+1}^{p_{k}}+f_{k}\right)
\end{aligned}
$$

$$
\begin{aligned}
& +\frac{1}{2}\left|\frac{\partial^{2} W_{k}}{\partial x_{0}^{2}}\right|\left|g_{0}\right|^{2}+\sum_{i=1}^{k-1} \frac{\partial W_{k}}{\partial x_{i}}\left(d_{i} u_{0}^{q_{i}} x_{i+1}^{p_{i}}+f_{i}\right) \\
& +\frac{1}{2}\left|\frac{\partial^{2} W_{k}}{\partial x_{k}^{2}}\right|\left|g_{k}\right|^{2}+\frac{1}{2} \sum_{i=1}^{k-1}\left|\frac{\partial^{2} W_{k}}{\partial x_{i}^{2}}\right|\left|g_{i}\right|^{2} \\
& +\frac{1}{2}\left|\frac{\partial^{2} W_{k}}{\partial x_{k} \partial x_{0}}\right|\left|g_{k}\right|\left|g_{0}^{\top}\right|+\frac{1}{2} \sum_{i=1}^{k-1}\left|\frac{\partial^{2} W_{k}}{\partial x_{i} \partial x_{0}}\right|\left|g_{i}\right|\left|g_{0}^{\top}\right| \\
& +\frac{1}{2} \sum_{i=1}^{k-1}\left|\frac{\partial^{2} W_{k}}{\partial x_{i} \partial x_{k}}\right|\left|g_{i}\right|\left|g_{k}^{\top}\right| \\
& +\frac{1}{2} \sum_{i, j=1, i \neq j}^{k-1}\left|\frac{\partial^{2} W_{k}}{\partial x_{i} \partial x_{j}}\right|\left|g_{i}\right|\left|g_{j}^{\top}\right| .
\end{aligned}
$$

We give the following proposition, whose proof is placed in the Appendix.

Proposition 18. There is a smooth function $\sigma_{k}$ such that

$$
\begin{aligned}
{\left[\xi_{k-1}\right]^{\left(4 l \sigma-\tau-r_{k-1}\right) / \sigma} d_{k-1} u_{0}^{q_{k-1}}\left(x_{k}^{p_{k-1}}-x_{k}^{* p_{k-1}}\right) } \\
+\frac{\partial W_{k}}{\partial x_{0}}\left(d_{0} u_{0}^{q_{0}}+f_{0}\right)+\sum_{i=1}^{k-1} \frac{\partial W_{k}}{\partial x_{i}}\left(d_{i} u_{0}^{q_{i}} x_{i+1}^{p_{i}}+f_{i}\right) \\
+\frac{\partial W_{k}}{\partial x_{k}} f_{k}+\frac{1}{2}\left|\frac{\partial^{2} W_{k}}{\partial x_{0}^{2}}\right|\left|g_{0}\right|^{2}+\frac{1}{2}\left|\frac{\partial^{2} W_{k}}{\partial x_{k}^{2}}\right|\left|g_{k}\right|^{2} \\
+\frac{1}{2} \sum_{i=1}^{k-1}\left|\frac{\partial^{2} W_{k}}{\partial x_{i} \partial x_{k}}\right|\left|g_{i}\right|\left|g_{k}^{\top}\right|+\frac{1}{2} \sum_{i=1}^{k-1}\left|\frac{\partial^{2} W_{k}}{\partial x_{i}^{2}}\right|\left|g_{i}\right|^{2} \\
+\frac{1}{2}\left|\frac{\partial^{2} W_{k}}{\partial x_{k} \partial x_{0}}\right|\left|g_{k}\right|\left|g_{0}^{\top}\right|+\frac{1}{2} \sum_{i=1}^{k-1}\left|\frac{\partial^{2} W_{k}}{\partial x_{i} \partial x_{0}}\right|\left|g_{i}\right|\left|g_{0}^{\top}\right| \\
+\frac{1}{2} \sum_{i, j=1, i \neq j}^{k-1}\left|\frac{\partial^{2} W_{k}}{\partial x_{i} \partial x_{j}}\right|\left|g_{i}\right|\left|g_{j}^{\top}\right| \\
+\bar{c}_{k-1} d_{k-1}\left|u_{0}\right|^{q_{k-1}}\left|\xi_{k-1}\right|^{\left(4 l \sigma-\tau-r_{k-1}\right) / \sigma}\left|\xi_{k}\right|^{\left(r_{k-1}+\tau\right) / \sigma} \\
\leq \frac{12}{13} \sum_{i=1}^{k-1} \xi_{i}^{4 l}+\sigma_{k} \Theta_{1} \xi_{k}^{4 l} \\
+\bar{c}_{k} d_{k}\left|u_{0}\right|^{q_{k}}\left|\xi_{k}\right|^{\left(4 l \sigma-\tau-r_{k}\right) / \sigma}\left|\xi_{k+1}\right|^{\left(r_{k}+\tau\right) / \sigma} \\
+\left|\xi_{k}\right|^{\left(4 l \sigma-\tau-r_{k}\right) / \sigma} d_{k} \bar{c}_{k}\left|u_{0}\right|^{q_{k}}\left|x_{k+1}^{*}\right|^{p_{k}},
\end{aligned}
$$

where $\bar{c}_{k}=2^{p_{k}-1} c_{k}$ is a nonnegative constant. Substituting (22) into (21), we arrive at

$$
\begin{aligned}
\mathscr{L} V_{k} \leq & -\left(n-k+\frac{14}{13}\right) \sum_{i=1}^{k-1} \xi_{i}^{4 l} \\
& +\left(\Psi_{k-1}-\dot{\hat{\Theta}}_{1}\right)\left(\widetilde{\Theta}_{1}+\eta_{k-1}\right)+\frac{\partial W_{k}}{\partial x_{k}} d_{k} u_{0}^{q_{k}} x_{k+1}^{p_{k}}
\end{aligned}
$$




$$
\begin{aligned}
& +\sigma_{k} \xi_{k}^{4 l} \Theta_{1}+\frac{\partial W_{k}}{\partial \widehat{\Theta}_{1}} \dot{\Theta}_{1} \\
& +\bar{c}_{k} d_{k}\left|u_{0}\right|^{q_{k}}\left|\xi_{k}\right|^{\left(4 l \sigma-\tau-r_{k}\right) / \sigma}\left|\xi_{k+1}\right|^{\left(r_{k}+\tau\right) / \sigma} \\
& +\left|\xi_{k}\right|^{\left(4 l \sigma-\tau-r_{k}\right) / \sigma} d_{k} \bar{c}_{k}\left|u_{0}\right|^{q_{k}}\left|x_{k+1}^{*}\right|^{p_{k}} .
\end{aligned}
$$

Obviously, defining $\Psi_{k}=\Psi_{k-1}+\xi_{k}^{4 l}\left(\sigma_{k}+n-k+14 / 13\right)$ and $\eta_{k}=\eta_{k-1}-\partial W_{k} / \partial \widehat{\Theta}_{1}$, it follows from (20) that $0 \leq \Psi_{k} \leq$ $\left(\xi_{1}^{4 l}+\cdots+\xi_{k}^{4 l}\right) \varphi_{k}$, where $\varphi_{k}=\max \left\{\varphi_{k-1}, \sigma_{k}+n-k+14 / 13\right\}$ is a nonnegative smooth function. With this in mind, (23) can be rewritten as

$$
\begin{aligned}
\mathscr{L} V_{k} \leq & -\left(n-k+\frac{14}{13}\right) \sum_{i=1}^{k} \xi_{i}^{4 l}+\left(\Psi_{k}-\dot{\widehat{\Theta}}_{1}\right)\left(\widetilde{\Theta}_{1}+\eta_{k}\right) \\
& +\frac{\partial W_{k}}{\partial x_{k}} d_{k} u_{0}^{q_{k}} x_{k+1}^{p_{k}} \\
& +\bar{c}_{k} d_{k}\left|u_{0}\right|^{q_{k}}\left|\xi_{k}\right|^{\left(4 l \sigma-\tau-r_{k}\right) / \sigma}\left|\xi_{k+1}\right|^{\left(r_{k}+\tau\right) / \sigma} \\
& +\left|\xi_{k}\right|^{\left(4 l \sigma-\tau-r_{k}\right) / \sigma} d_{k} \bar{c}_{k}\left|u_{0}\right|^{q_{k}}\left|x_{k+1}^{*}\right|^{p_{k}} \\
& +\xi_{k}^{4 l} \widehat{\Theta}_{1}\left(\sigma_{k}+n-k+\frac{14}{13}\right)+\eta_{k-1} \Psi_{k-1} \\
& -\eta_{k} \Psi_{k} .
\end{aligned}
$$

With the help of Lemma 4, one has

$$
\begin{aligned}
\left|\eta_{k-1} \Psi_{k-1}-\eta_{k} \Psi_{k}\right| \leq & \left|\eta_{k-1} \xi_{k}^{4 l} \widehat{\Theta}_{1}\left(\sigma_{k}+n-k+\frac{14}{13}\right)\right| \\
& +\left|\frac{\partial W_{k}}{\partial \widehat{\Theta}_{1}} \Psi_{k}\right| \\
\leq & \frac{1}{13} \sum_{i=1}^{k} \xi_{i}^{4 l}+\bar{\varphi}_{k} \widehat{\Theta}_{1} \xi_{k}^{4 l},
\end{aligned}
$$

which, together with (24), implies that

$$
\begin{aligned}
& \mathscr{L} V_{k} \leq-(n-k+1) \sum_{i=1}^{k} \xi_{i}^{4 l}+\left(\Psi_{k}-\dot{\widehat{\Theta}}_{1}\right)\left(\widetilde{\Theta}_{1}+\eta_{k}\right) \\
& +\left\lceil\xi_{k}\right\rceil^{\left(4 l \sigma-\tau-r_{k}\right) / \sigma} d_{k} u_{0}^{q_{k}}\left(x_{k+1}^{p_{k}}-x_{k+1}^{* p_{k}}\right) \\
& +\left\lceil\xi_{k}\right\rceil^{\left(4 l \sigma-\tau-r_{k}\right) / \sigma}\left(d_{k} u_{0}^{q_{k}}\left(1-\bar{c}_{k}\right) x_{k+1}^{* p_{k}}+\left\lceil\xi_{k}\right\rceil^{\left(r_{k}+\tau\right) / \sigma}\right. \\
& \left.\cdot \widehat{\Theta}_{1}\left(\bar{\varphi}_{k}+\sigma_{k}+n-k+\frac{14}{13}\right)\right)+\bar{c}_{k} d_{k}\left|u_{0}\right|^{q_{k}} \\
& \cdot\left|\xi_{k}\right|^{\left(4 l \sigma-\tau-r_{k}\right) / \sigma}\left|\xi_{k+1}\right|^{\left(r_{k}+\tau\right) / \sigma}
\end{aligned}
$$

where $\bar{\varphi}_{k} \geq 0$ is a smooth function. It is easy to see that the virtual controller

$$
x_{k+1}^{*}=-\left(\frac{1}{\lambda_{k, 1} u_{0}^{q_{k}}\left(1-\bar{c}_{k}\right)}\right.
$$

$$
\begin{aligned}
& \left.\cdot \widehat{\Theta}_{1}\left(\bar{\varphi}_{k}+\sigma_{k}+n-k+\frac{14}{13}\right)\right)^{1 / p_{k}}\left\lceil\xi_{k}\right\rceil^{r_{k+1} / \sigma} \\
& \triangleq-\phi_{k}\left(x_{0}, \bar{x}_{k}, \widehat{\Theta}_{1}\right)\left\lceil\xi_{k}\right\rceil^{r_{k+1} / \sigma},
\end{aligned}
$$

renders

$$
\begin{aligned}
\mathscr{L} V_{k} \leq & -(n-k+1) \sum_{i=1}^{k} \xi_{i}^{4 l} \\
& +\left\lceil\left.\xi_{k}\right|^{\left(4 l \sigma-\tau-r_{k}\right) / \sigma} d_{k} u_{0}^{q_{k}}\left(x_{k+1}^{p_{k}}-x_{k+1}^{* p_{k}}\right)\right. \\
& +\left(\Psi_{k}-\dot{\widehat{\Theta}}_{1}\right)\left(\widetilde{\Theta}_{1}+\eta_{k}\right) \\
& +\bar{c}_{k} d_{k}\left|u_{0}\right|^{q_{k}}\left|\xi_{k}\right|^{\left(4 l \sigma-\tau-r_{k}\right) / \sigma}\left|\xi_{k+1}\right|^{\left(r_{k}+\tau\right) / \sigma},
\end{aligned}
$$

which still holds for $k=n$. Hence at last step, we can explicitly construct a positive-definite and proper Lyapunov function $V_{n}=V_{n-1}+W_{n}$ and a smooth controller $x_{n+1}^{*}$ with form (27) such that

$$
\begin{aligned}
\mathscr{L} V_{n} \leq & -\sum_{i=1}^{n} \xi_{i}^{4 l}+\left(\Psi_{n}-\dot{\widehat{\Theta}}_{1}\right)\left(\widetilde{\Theta}_{1}+\eta_{n}\right) \\
& +\left\lceil\left.\xi_{n}\right|^{\left(4 l \sigma-\tau-r_{n}\right) / \sigma} d_{n} u_{0}^{q_{n}}\left(u^{p_{n}}-x_{n+1}^{* p_{n}}\right)\right. \\
& +\bar{c}_{n} d_{n}\left|u_{0}\right|^{q_{n}}\left|\xi_{n}\right|^{\left(4 l \sigma-\tau-r_{n}\right) / \sigma}\left|\xi_{n+1}\right|^{\left(r_{n}+\tau\right) / \sigma} .
\end{aligned}
$$

Noting $\xi_{n+1}=0$, by choosing the smooth actual controller $u$ and the adaptive law for $\widehat{\Theta}_{1}$,

$$
u=x_{n+1}^{*}=-\phi_{n}\left(x_{0}, \bar{x}_{n}, \widehat{\Theta}_{1}\right)\left\lceil\xi_{n}\right\rceil^{r_{n+1} / \sigma}, \quad \dot{\widehat{\Theta}}_{1}=\Psi_{n},
$$

we get

$$
\mathscr{L} V_{n} \leq-\sum_{i=1}^{n} \xi_{i}^{4 l}
$$

The succeeding proposition characterizes the features of $x_{i}$ subsystem.

Proposition 19. If Assumptions 9-11 hold for system (1), controllers (11) and (30) guarantee that

(i) the closed-loop system has an almost surely unique solution on $\left[t_{0}, \infty\right)$ for each $\left(x_{1}\left(t_{0}\right), \ldots, x_{n}\left(t_{0}\right), \widehat{\Theta}_{1}\left(t_{0}\right)\right)$

(ii) the equilibrium of the closed-loop system is globally asymptotically stable in probability, $P\left\{\lim _{t \rightarrow \infty}|x(t)|=\right.$ $0\}=1, P\left\{\lim _{t \rightarrow \infty} \widehat{\Theta}_{1}(t)\right.$ exist and is finite $\}=1$ for each $\left(x_{1}\left(t_{0}\right), \ldots, x_{n}\left(t_{0}\right), \widehat{\Theta}_{1}\left(t_{0}\right)\right)$

Proof. See Appendix.

Case II $\left(x_{0}\left(t_{0}\right)=0\right)$. When $x_{0}\left(t_{0}\right)=0$, how to select the controllers $u_{0}$ and $u$ is an interesting topic. If the initial state is zero, one chooses an open loop controller $u_{0}=u_{0}^{*} \neq 0$ to drive the state $x_{0}$ away from zero; a novel controller $u=$ $u^{*}$ can be obtained by the above procedure of original $x_{i}$ subsystem in (1). So there exists $t_{s}>0$ such that $x_{0}\left(t_{s}\right) \neq 0$; after that, controllers $u_{0}$ in (11) and $u$ in (30) can be used. 
In view of the argument above, there exists an adaptive controller, such that the equilibrium of the closed-loop system is globally asymptotically stable in probability for any initial condition. The proof of Theorem 15 is completed.

\section{Simulation Example}

Consider the bilinear model of a mobile robot with small angle measurement error [26], which is described by

$$
\begin{aligned}
& \dot{x}_{c}=\left(1-\frac{\varepsilon^{2}}{2}\right) v, \\
& \dot{y}_{c}=\theta v+\varepsilon v, \\
& \dot{\theta}=\omega,
\end{aligned}
$$

where $\varepsilon$ is a small bias in orientation. $\nu$ and $\omega$ are two control inputs to denote the linear velocity and angular velocity, respectively. Since stochastic disturbance frequently arises in practical control systems, when the angular velocity $\omega$ is subject to some stochastic disturbances, $\omega$ can be expressed as $\omega\left(x_{c}, y_{c}, \theta\right)=\omega_{1}\left(x_{c}, y_{c}, \theta\right)+\omega_{2}\left(x_{c}, y_{c}, \theta\right) \dot{B}(t)$, where $B(t)$ is the so-called white noise [2]. Then system (32) is transformed into

$$
\begin{aligned}
\mathrm{d} x_{c} & =\left(1-\frac{\varepsilon^{2}}{2}\right) \nu \mathrm{d} t, \\
\mathrm{~d} y_{c} & =(\theta \nu+\varepsilon v) \mathrm{d} t, \\
\mathrm{~d} \theta & =\omega_{1} \mathrm{~d} t+\omega_{2} \mathrm{~d} B .
\end{aligned}
$$

For system (33), by taking the state and input transformation $x_{0}=x_{c}, x_{1}=y_{c}, x_{2}=\theta+\varepsilon, u_{0}=v, u_{1}=\omega_{1}$, one has

$$
\begin{aligned}
& \mathrm{d} x_{0}=\left(1-\frac{\varepsilon^{2}}{2}\right) u_{0} \mathrm{~d} t \\
& \mathrm{~d} x_{1}=x_{2} u_{0} \mathrm{~d} t \\
& \mathrm{~d} x_{2}=u_{1} \mathrm{~d} t+\omega_{2} \mathrm{~d} B .
\end{aligned}
$$

System (34) is a special case of system (1). For simplicity, we assume $1-\varepsilon^{2} / 2>0, \omega_{2}=x_{2} \sin x_{1}$, and $x_{0}(0) \neq 0$.
Following the above design process, an adaptive controller can be explicitly given

$$
\begin{aligned}
& u_{0}=-x_{0}, \\
& u_{1}=-\widehat{\Theta}_{1}\left(\bar{\varphi}_{2}+\sigma+\frac{14}{13}\right)\left\lceil\xi_{1}\right\rceil, \\
& \dot{\Theta}_{1}=2 \xi_{1}^{4}+\xi_{2}^{4}\left(\sigma+\frac{14}{13}\right),
\end{aligned}
$$

where $\xi_{1}=\left\lceil x_{1}\right\rceil, \xi_{2}=\left\lceil x_{2}\right\rceil-2 \widehat{\Theta}_{1} u_{0}^{-1}\left\lceil\xi_{1}\right\rceil, \bar{\varphi}_{2}=$ $12\left|u_{0}\right|^{-4 / 3}(\sigma+14 / 13)^{-4 / 3}\left(\xi_{1}^{4}+\xi_{2}^{4}\right), \sigma=231.7 u_{0}^{4} \widetilde{\Theta}_{1}^{3}+$ $6 \widehat{\Theta}_{1}+12 \widehat{\Theta}_{1}^{4 / 3}+231.7$. By choosing $\varepsilon=1$ and initial values $\left(x_{0}(0), x_{1}(0), x_{2}(0), \widehat{\Theta}_{1}(0)\right)=(2,-0.25,2,1)$, Figure 1 demonstrates the effectiveness of the control scheme.

\section{Conclusions}

This paper investigates adaptive state feedback stabilization for more general stochastic high-order nonholonomic systems. There still exist some problems to be investigated; for instance, we have the following. (1) The result in this paper can be applied to the case of $p_{i} \in R_{o d d}^{\geq 1}$. However, if $p_{i}$ is an even number or a ratio of odd integer and even integer, it is unclear whether the control strategy can be applied or not. (2) Recently, some results on stochastic nonlinear systems with SiISS dynamic uncertainty have been obtained [27-35]. An important problem is how to solve adaptive feedback control for stochastic nonholonomic nonlinear systems with SiISS dynamic and parametric uncertainties.

\section{Appendix}

Proof of Proposition 16. It is not hard to prove that $\widetilde{\Theta}_{0}$ is bounded. By using (12) and Lemma 2, the equilibrium $x_{0}(t)=0$ of $x_{0}$-subsystem is globally stable in probability and $P\left\{\lim _{t \rightarrow \infty}\left|x_{0}(t)=0\right|\right\}=1$ for any $x_{0}\left(t_{0}\right) \neq 0$. By Definition 1 , the equilibrium $x_{0}(t)=0$ of $x_{0}$-subsystem is globally asymptotically stable in probability. Substituting (11) into (1), it is easy to obtain

$$
\begin{aligned}
\mathrm{d} x_{0}= & \left(-d_{0} h\left(x_{0}\right) \sqrt{\widehat{\Theta}_{0}^{2}+1}+\alpha_{0}\left(x_{0}, \theta\right)\right) x_{0} \mathrm{~d} t \\
& +\beta_{0}^{\top}\left(x_{0}, \theta\right) x_{0} \mathrm{~d} \omega,
\end{aligned}
$$

whose solution is

$$
\begin{aligned}
& x_{0}(t)=x_{0}\left(t_{0}\right) \exp \left(\int_{t_{0}}^{t}\left(-d_{0}(s, \theta) h\left(x_{0}(s)\right) \sqrt{\widehat{\Theta}_{0}^{2}+1}+\alpha_{0}\left(x_{0}(s), \theta\right)-\frac{1}{2} \beta_{0}^{\top}\left(x_{0}(s), \theta\right) \beta_{0}\left(x_{0}(s), \theta\right)\right) \mathrm{d} s\right. \\
& \left.\quad+\int_{t_{0}}^{t} \beta_{0}^{\top}\left(x_{0}(s), \theta\right) \mathrm{d} \omega\right) .
\end{aligned}
$$

By using Lemma 2.3 in [2], for any $x_{0}\left(t_{0}\right) \neq 0$, there holds $x_{0}(t) \neq 0$ for any $t \geq t_{0}$, which implies that $u_{0}$ does not cross zero. The proof of Proposition 16 is completed. 

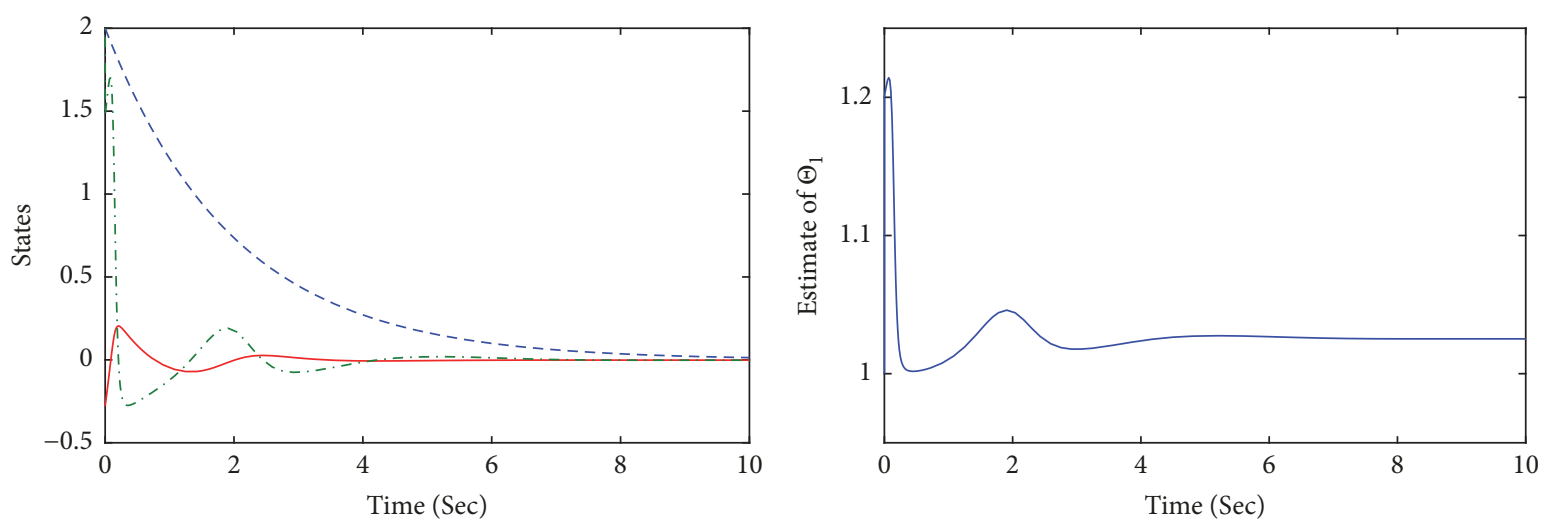

$$
\begin{aligned}
-- & x_{0} \\
- & x_{1} \\
-\ldots & x_{2}
\end{aligned}
$$
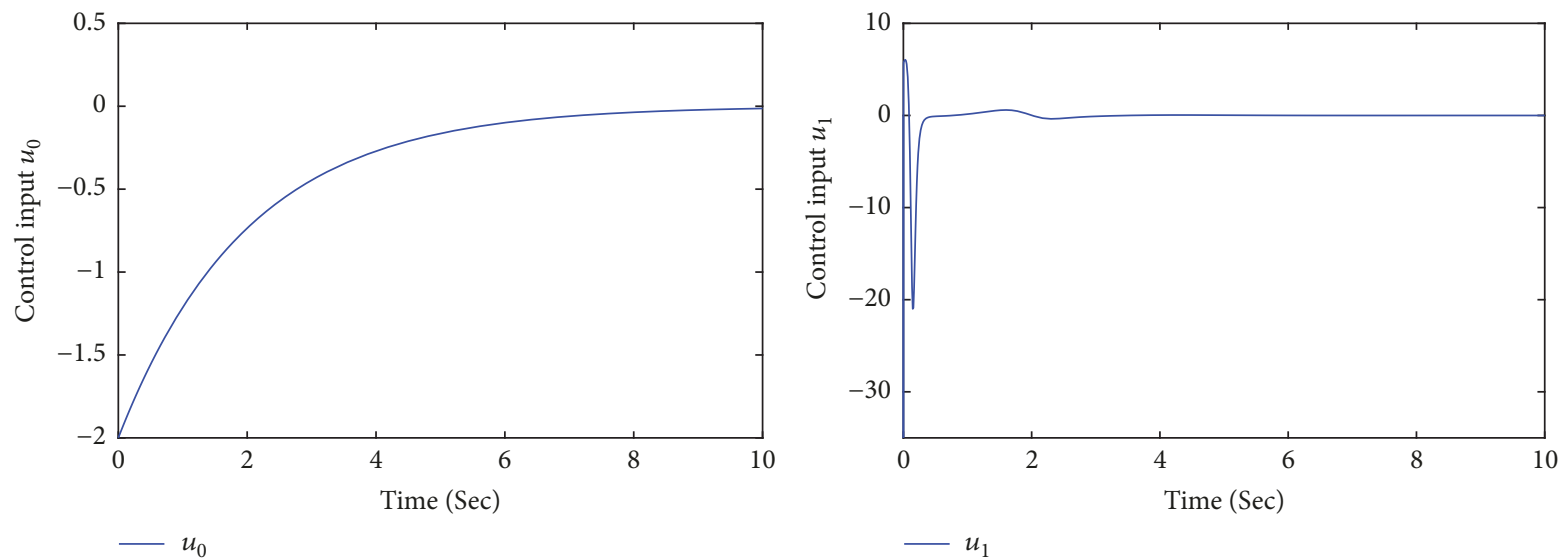

FIGURE 1: Trajectories of $x_{0}, x_{1}, x_{2}, \widehat{\Theta}_{1}, u_{0} u_{1}$.

Proof of Proposition 17. From (14) and Lemma 5, we obtain

$$
\begin{aligned}
& \frac{\partial W_{k}}{\partial x_{k}}=\left\lceil\xi_{k}\right\rceil^{\left(4 l \sigma-\tau-r_{k}\right) / \sigma} \\
& \frac{\partial W_{k}}{\partial x_{i}}=-\frac{4 l \sigma-\tau-r_{k}}{\sigma} \frac{\partial\left(\left\lceil x_{k}^{*}\right\rceil^{\sigma / r_{k}}\right)}{\partial x_{i}} \\
& \cdot \int_{x_{k}^{*}}^{x_{k}}\left|\lceil s\rceil^{\sigma / r_{k}}-\left\lceil x_{k}^{*}\right\rceil^{\sigma / r_{k}}\right|^{\left((4 l-1) \sigma-\tau-r_{k}\right) / \sigma} \mathrm{d} s,
\end{aligned}
$$

where $i=1, \ldots, k-1$. Exactly following the same procedure as in the proof of (A.4), we get

$$
\begin{gathered}
\frac{\partial W_{k}}{\partial \widehat{\Theta}_{1}}=-\frac{4 l \sigma-\tau-r_{k}}{\sigma} \frac{\partial\left(\left\lceil x_{k}^{*}\right\rceil^{\sigma / r_{k}}\right)}{\partial \widehat{\Theta}_{1}} \\
\cdot \int_{x_{k}^{*}}^{x_{k}}\left|\lceil s\rceil^{\sigma / r_{k}}-\left\lceil x_{k}^{*}\right\rceil^{\sigma / r_{k}}\right|^{\left((4 l-1) \sigma-\tau-r_{k}\right) / \sigma} \mathrm{d} s, \\
\frac{\partial W_{k}}{\partial x_{0}}=-\frac{4 l \sigma-\tau-r_{k}}{\sigma} \frac{\partial\left(\left\lceil x_{k}^{*}\right\rceil^{\sigma / r_{k}}\right)}{\partial x_{0}} \\
\cdot \int_{x_{k}^{*}}^{x_{k}}\left|\lceil s\rceil^{\sigma / r_{k}}-\left\lceil x_{k}^{*}\right\rceil^{\sigma / r_{k}}\right|^{\left((4 l-1) \sigma-\tau-r_{k}\right) / \sigma} \mathrm{d} s .
\end{gathered}
$$

By using (A.3), (A.4), and Lemma 5,

$$
\begin{aligned}
& \frac{\partial^{2} W_{k}}{\partial x_{k}^{2}}=\frac{4 l \sigma-\tau-r_{k}}{r_{k}}\left|\xi_{k}\right|^{\left((4 l-1) \sigma-\tau-r_{k}\right) / \sigma}\left|x_{k}\right|^{\left(\sigma-r_{k}\right) / r_{k}}, \\
& \frac{\partial^{2} W_{k}}{\partial x_{i}^{2}}=\left(\frac{(4 l-1) \sigma-\tau-r_{k}}{\sigma}\right.
\end{aligned}
$$

$$
\begin{aligned}
& \cdot \int_{x_{k}^{*}}^{x_{k}}\left|\lceil s\rceil^{\sigma / r_{k}}-\left\lceil x_{k}^{*}\right\rceil^{\sigma / r_{k}}\right|^{\left((4 l-2) \sigma-\tau-r_{k}\right) / \sigma} \mathrm{d} s\left(\frac{\partial\left(\left\lceil x_{k}^{*}\right\rceil^{\sigma / r_{k}}\right)}{\partial x_{i}}\right)^{2} \\
& \left.-\int_{x_{k}^{*}}^{x_{k}}\left|\lceil s\rceil^{\sigma / r_{k}}-\left\lceil x_{k}^{*}\right\rceil^{\sigma / r_{k}}\right|^{\left((4 l-1) \sigma-\tau-r_{k}\right) / \sigma} \mathrm{d} s \frac{\partial^{2}\left(\left\lceil x_{k}^{*}\right\rceil^{\sigma / r_{k}}\right)}{\partial x_{i}^{2}}\right) \\
& \cdot \frac{4 l \sigma-\tau-r_{k}}{\sigma} .
\end{aligned}
$$

Similar to (A.8), it follows that

$$
\frac{\partial^{2} W_{k}}{\partial x_{0}^{2}}=\left(\frac{(4 l-1) \sigma-\tau-r_{k}}{\sigma}\right.
$$




$$
\begin{aligned}
& \cdot \int_{x_{k}^{*}}^{x_{k}}\left|\lceil s\rceil^{\sigma / r_{k}}-\left\lceil x_{k}^{*}\right\rceil^{\sigma / r_{k}}\right|^{\left((4 l-2) \sigma-\tau-r_{k}\right) / \sigma} \mathrm{d} s\left(\frac{\partial\left(\left\lceil x_{k}^{*}\right\rceil^{\sigma / r_{k}}\right)}{\partial x_{0}}\right)^{2} \\
& \left.-\int_{x_{k}^{*}}^{x_{k}}\left|\lceil s\rceil^{\sigma / r_{k}}-\left\lceil x_{k}^{*}\right\rceil^{\sigma / r_{k}}\right|^{\left((4 l-1) \sigma-\tau-r_{k}\right) / \sigma} \mathrm{d} s \frac{\partial^{2}\left(\left\lceil x_{k}^{*}\right\rceil^{\sigma / r_{k}}\right)}{\partial x_{0}^{2}}\right)
\end{aligned}
$$

Moreover, it is clear that

$$
\begin{aligned}
& \frac{\partial^{2} W_{k}}{\partial x_{i} \partial x_{k}}=\frac{\partial^{2} W_{k}}{\partial x_{k} \partial x_{i}}=-\frac{4 l \sigma-\tau-r_{k}}{\sigma}\left|\xi_{k}\right| \frac{(4 l-1) \sigma-\tau-r_{k}}{\sigma} \frac{\partial\left(\left\lceil x_{k}^{*}\right]^{\sigma / r_{k}}\right)}{\partial x_{i}}, \\
& \frac{\partial^{2} W_{k}}{\partial x_{0} \partial x_{k}}=\frac{\partial^{2} W_{k}}{\partial x_{k} \partial x_{0}}=-\frac{4 l \sigma-\tau-r_{k}}{\sigma}\left|\xi_{k}\right|^{\left((4 l-1) \sigma-\tau-r_{k}\right) / \sigma} \frac{\partial\left(\left\lceil x_{k}^{*}\right\rceil^{\sigma / r_{k}}\right)}{\partial x_{0}} \\
& \frac{\partial^{2} W_{k}}{\partial x_{i} \partial x_{0}}=\frac{\partial^{2} W_{k}}{\partial x_{0} \partial x_{i}}=\left(\frac{(4 l-1) \sigma-\tau-r_{k}}{\sigma} \int_{x_{\mathrm{k}}^{*}}^{x_{k}}\left|\lceil s\rceil^{\sigma / r_{k}}-\left\lceil x_{k}^{*}\right\rceil^{\sigma / r_{k}}\right|^{\left((4 l-2) \sigma-\tau-r_{k}\right) / \sigma} \mathrm{d} s \frac{\partial\left(\left\lceil x_{k}^{*}\right\rceil^{\sigma / r_{k}}\right)}{\partial x_{i}} \frac{\partial\left(\left\lceil x_{k}^{*}\right\rceil^{\sigma / r_{k}}\right)}{\partial x_{0}}\right. \\
& \left.-\int_{x_{k}^{*}}^{x_{k}}\left|\lceil s\rceil^{\sigma / r_{k}}-\left\lceil x_{k}^{*}\right\rceil^{\sigma / r_{k}}\right|^{\left((4 l-1) \sigma-\tau-r_{k}\right) / \sigma} \mathrm{d} s \frac{\partial^{2}\left(\left\lceil x_{k}^{*}\right\rceil^{\left(\sigma / r_{k}\right)}\right)}{\partial x_{i} \partial x_{0}}\right) \frac{4 l \sigma-\tau-r_{k}}{\sigma} \\
& \frac{\partial^{2} W_{k}}{\partial x_{i} \partial x_{j}}=\frac{\partial^{2} W_{k}}{\partial x_{j} \partial x_{i}}=\left(\frac{(4 l-1) \sigma-\tau-r_{k}}{\sigma} \int_{x_{k}^{*}}^{x_{k}}\left|\lceil s\rceil^{\sigma / r_{k}}-\left\lceil x_{k}^{*}\right\rceil^{\sigma / r_{k}}\right|^{\left((4 l-2) \sigma-\tau-r_{k}\right) / \sigma} \mathrm{d} s \frac{\partial\left(\left\lceil x_{k}^{*}\right\rceil^{\sigma / r_{k}}\right)}{\partial x_{i}} \frac{\partial\left(\left\lceil x_{k}^{*}\right\rceil^{\sigma / r_{k}}\right)}{\partial x_{j}}\right. \\
& \left.-\int_{x_{k}^{*}}^{x_{k}}\left|\lceil s\rceil^{\sigma / r_{k}}-\left\lceil x_{k}^{*}\right\rceil^{\sigma / r_{k}}\right|^{\left((4 l-1) \sigma-\tau-r_{k}\right) / \sigma} \mathrm{d} s \frac{\partial^{2}\left(\left\lceil x_{k}^{*}\right\rceil^{\sigma / r_{k}}\right)}{\partial x_{i} \partial x_{j}}\right) \frac{4 l \sigma-\tau-r_{k}}{\sigma},
\end{aligned}
$$

where $j=1, \ldots, k-1$ and $j \neq i$. From the expression of $x_{k}^{*}$, we know that $\partial\left(\left\lceil x_{k}^{*}\right\rceil^{\sigma / r_{k}}\right) / \partial x_{i}, \partial^{2}\left(\left\lceil x_{k}^{*}\right\rceil^{\sigma / r_{k}}\right) / \partial x_{i}^{2}$, $\partial^{2}\left(\left\lceil x_{k}^{*}\right\rceil^{\sigma / r_{k}}\right) / \partial x_{i} \partial x_{j}, \quad \partial^{2}\left(\left\lceil x_{k}^{*}\right\rceil^{\sigma / r_{k}}\right) / \partial x_{i} \partial x_{0}, \quad \partial\left(\left\lceil x_{k}^{*}\right\rceil^{\sigma / r_{k}}\right) / \partial x_{0}$, $\partial\left(\left\lceil x_{k}^{*}\right\rceil^{\sigma / r_{k}}\right) / \partial \widehat{\Theta}_{1}$ are continuous functions. It is straightforward to deduce the conclusion that $W_{k}\left(x_{0}, \bar{x}_{k}, \widehat{\Theta}_{1}\right)$ is $\mathscr{C}^{2}$ function.

The rest of proof is divided into two steps. Firstly, we prove the right side of inequality in Proposition 17. In view of Lemmas 6 and 8, one concludes that

$$
\begin{aligned}
& W_{k}\left(x_{0}, \bar{x}_{k}, \widehat{\Theta}_{1}\right) \\
& \quad \leq\left|\xi_{k}\right|^{\left(4 l \sigma-\tau-r_{k}\right) / \sigma} 2^{1-r_{k} / \sigma} \mid\left\lceil\left. x_{k}\right|^{\sigma / r_{k}}-\left.\left\lceil x_{k}^{*}\right\rceil^{\sigma / r_{k}}\right|^{r_{k} / \sigma}\right. \\
& \quad=2^{1-r_{k} / \sigma}\left|\xi_{k}\right|^{(4 l \sigma-\tau) / \sigma} .
\end{aligned}
$$

The next work is to prove the left side of inequality. The issue can be solved by considering two different cases. If $x_{k} \geq x_{k}^{*}$, then $\lceil s\rceil^{\sigma / r_{k}}-\left\lceil x_{k}^{*}\right\rceil^{\sigma / r_{k}} \geq 0$, where $x_{k} \geq s \geq x_{k}^{*}$. By Lemma 6, we obtain

$$
\begin{aligned}
& W_{k}\left(x_{0}, \bar{x}_{k}, \widehat{\Theta}_{1}\right) \\
& \quad \geq \int_{x_{k}^{*}}^{x_{k}}\left(2^{r_{k} / \sigma-1}\left(s-x_{k}^{*}\right)\right)^{\left(4 l \sigma-\tau-r_{k}\right) / r_{k}} \mathrm{~d} s \\
& \quad=2^{\left(r_{k}-\sigma\right)\left(4 l \sigma-\tau-r_{k}\right) / \sigma r_{k}} \frac{r_{k}}{4 l \sigma+q_{k}}\left(x_{k}-x_{k}^{*}\right)^{(4 l \sigma-\tau) / r_{k}} .
\end{aligned}
$$

If $x_{k}<x_{k}^{*}$, in a similar way,

$$
\begin{aligned}
& W_{k}\left(x_{0}, \bar{x}_{k}, \widehat{\Theta}_{1}\right) \\
& \quad \geq 2^{\left(r_{k}-\sigma\right)\left(4 l \sigma-\tau-r_{k}\right) / \sigma r_{k}} \frac{r_{k}}{4 l \sigma-\tau}\left(x_{k}^{*}-x_{k}\right)^{(4 l \sigma-\tau) / r_{k}} .
\end{aligned}
$$

Combining (A.15) with (A.16), the proof of Proposition 17 is completed.

Proof of Proposition 18. Since $p_{k-1}, k=2,3, \ldots, n+1$ are odd integers, by using Lemma 6,

$$
\left|x_{k}^{p_{k-1}}-x_{k}^{* p_{k-1}}\right| \leq 2^{1-r_{k} p_{k-1} / \sigma}\left|\xi_{k}\right|^{r_{k} p_{k-1} / \sigma} .
$$

According to (A.17), Remark 14, and Lemma 4, it follows that

$$
\begin{aligned}
& \left\lceil\xi_{k-1}\right\rceil^{\left(4 l \sigma-\tau-r_{k-1}\right) / \sigma} d_{k-1} u_{0}^{q_{k-1}}\left(x_{k}^{p_{k-1}}-x_{k}^{* p_{k-1}}\right) \\
& \leq \frac{1}{13} \xi_{k-1}^{4 l}+\sigma_{k 1} \Theta_{1} \xi_{k}^{4 l},
\end{aligned}
$$

where $\sigma_{k 1}$ is a positive constant. It is easy to deduce from (13) that

$$
\left|\frac{\partial\left(\left\lceil x_{k}^{*}\right\rceil^{\sigma / r_{k}}\right)}{\partial x_{0}}\right| \leq\left|\xi_{k-1}\right|\left|\frac{\partial \phi_{k-1}^{\sigma / r_{k}}}{\partial x_{0}}\right|
$$




$$
\begin{aligned}
& +\left|\phi_{k-1}\right|^{\sigma / r_{k}}\left|\frac{\partial\left(-\left\lceil x_{k-1}^{*}\right\rceil^{\sigma / r_{k-1}}\right)}{\partial x_{0}}\right| \\
& \vdots \\
& \leq \sum_{j=1}^{k-2} \prod_{l=j+1}^{k-1}\left|\phi_{l}\right|^{\sigma / r_{l+1}}\left|\frac{\partial \phi_{j}^{\sigma / r_{j+1}}}{\partial x_{0}}\right|\left|\xi_{j}\right|+\left|\xi_{k-1}\right|\left|\frac{\partial \phi_{k-1}^{\sigma / r_{k}}}{\partial x_{0}}\right| \\
& \leq \sum_{j=1}^{k-1} \psi_{j, 1}\left|\xi_{j}\right|,
\end{aligned}
$$

where $\psi_{j, 1}=\max \left\{\left(\prod_{l=j}^{k-1}\left|\phi_{l}\right|^{\sigma / r_{l+1}}\right)\left|\partial \phi_{j}^{\sigma / r_{j+1}} / \partial x_{0}\right|,\left|\partial \phi_{k-1}^{\sigma / r_{k}} / \partial x_{0}\right|\right\}$, $j=1, \ldots, k-1$, are continuous functions. It follows from (A.6), (A.19), Remark 14, and Lemmas 4 and 8 that

$$
\begin{aligned}
& \frac{\partial W_{k}}{\partial x_{0}}\left(d_{0} u_{0}^{p_{0}}+f_{0}\right) \leq\left(\left|d_{0} u_{0}^{p_{0}}\right|+\left|f_{0}\right|\right)\left|\xi_{k}\right|^{-\tau / \sigma} 2^{1-r_{k} / \sigma} \\
& \cdot \frac{4 l \sigma-\tau-r_{k}}{\sigma}\left|\xi_{k}\right|^{(4 l-1) \sigma / \sigma} \sum_{j=1}^{k-1} \psi_{j, 1}\left|\xi_{j}\right| \leq \frac{1}{13} \xi_{k-1}^{4 l} \\
& +\sigma_{k 2} \Theta_{1} \xi_{k}^{4 l},
\end{aligned}
$$

where $\sigma_{k 2} \geq 0$ is a smooth function. According to (13) and (A.19), we arrive at

$$
\begin{aligned}
& \left|\frac{\partial^{2}\left(\left\lceil x_{k}^{*}\right\rceil^{\sigma / r_{k}}\right)}{\partial x_{0}^{2}}\right| \\
& \leq\left|\frac{\partial^{2} \phi_{k-1}^{\sigma / r_{k}}}{\partial x_{0}^{2}}\right|\left|\xi_{k-1}\right|+\left|\frac{\partial \phi_{k-1}^{\sigma / r_{k}}}{\partial x_{0}}\right|\left|\frac{\partial\left(\left\lceil x_{k}^{*}\right\rceil^{\sigma / r_{k}}\right)}{\partial x_{0}}\right| \\
& \quad+\sum_{j=1}^{k-2} \frac{\partial\left(\prod_{l=j+1}^{k-1}\left|\phi_{l}\right|^{\sigma / r_{l+1}}\left|\partial \phi_{j}^{\sigma / r_{j+1}} / \partial x_{0}\right|\right)}{\partial x_{0}}\left|\xi_{j}\right| \\
& \quad+\sum_{j=1}^{k-2} \prod_{l=j+1}^{k-1}\left|\phi_{l}\right|^{\sigma / r_{l+1}}\left|\frac{\partial \phi_{j}^{\sigma / r_{j+1}}}{\partial x_{0}}\right|\left|\frac{\left.\partial\left(\mid x_{j}^{*}\right]^{\sigma / r_{j}}\right)}{\partial x_{0}}\right| \\
& \leq \sum_{j=1}^{k-1} \psi_{j, 2}\left|\xi_{j}\right|,
\end{aligned}
$$

where $\psi_{j, 2} \geq 0, j=1, \ldots, k-1$, are continuous functions. By using (A.9), (A.19), (A.21), Remark 14, and Lemmas 4 and 8, one has

$$
\begin{aligned}
& \frac{1}{2}\left|\frac{\partial^{2} W_{k}}{\partial x_{0}^{2}}\right|\left|g_{0}\right|^{2} \leq 2^{-r_{k} / \sigma} \frac{4 l \sigma-\tau-r_{k}}{\sigma} \\
& \cdot \bar{\beta}_{0}^{2} \Theta_{1} x_{0}^{2}\left(\left|\xi_{k}\right|^{-\tau / \sigma} \frac{(4 l-1) \sigma-\tau-r_{k}}{\sigma}\left|\xi_{k}\right|^{(4 l-2) \sigma / \sigma}\right.
\end{aligned}
$$

$$
\begin{aligned}
& \left(\sum_{j=1}^{k-1} \psi_{j, 1}\left|\xi_{j}\right|\right)^{2}+\left|\xi_{k}\right|^{-\tau / \sigma}\left|\xi_{k}\right|^{(4 l-1) \sigma / \sigma} \\
& \left.\cdot \sum_{j=1}^{k-1} \psi_{j, 3}\left|\xi_{j}\right|\right) \leq \frac{1}{13} \xi_{k-1}^{4 l}+\sigma_{k 3} \Theta_{1} \xi_{k}^{4 l}
\end{aligned}
$$

where $\sigma_{k 3} \geq 0$ is a smooth function. From (13) and Lemma 7, one arrives at

$$
\begin{aligned}
& \sum_{i=1}^{k}\left|x_{i}\right|^{\left(r_{k}+\tau\right) / r_{i}} \\
& \quad \leq \sum_{i=1}^{k}\left(\left|\xi_{i}\right|^{\left(r_{k}+\tau\right) / \sigma}+\phi_{i-1}^{\left(r_{k}+\tau\right) / r_{i}}\left|\xi_{i-1}\right|^{\left(r_{k}+\tau\right) / \sigma}\right), \\
& \sum_{i=1}^{k}\left|x_{i}\right|^{\left(2 r_{k}+\tau\right) / 2 r_{i}} \\
& \quad \leq \sum_{i=1}^{k}\left(\left|\xi_{i}\right|^{\left(2 r_{k}+\tau\right) / 2 \sigma}+\phi_{i-1}^{\left(2 r_{k}+\tau\right) / 2 r_{i}}\left|\xi_{i-1}\right|^{\left(2 r_{k}+\tau\right) / 2 \sigma}\right)
\end{aligned}
$$

where $\phi_{0}=0$ and $\xi_{0}=0$. By (A.3), (A.23) and Lemma 4 , it is easy to verify that

$$
\begin{aligned}
& \frac{\partial W_{k}}{\partial x_{k}} f_{k} \leq\left\lceil\xi_{k}\right]^{\left(4 l \sigma-\tau-r_{k}\right) / \sigma} \\
& \quad \cdot \sum_{i=1}^{k}\left(\left|\xi_{i}\right|^{\left(r_{k}+\tau\right) / \sigma}+\phi_{i-1}^{\left(r_{k}+\tau\right) / r_{i}}\left|\xi_{i-1}\right|^{\left(r_{k}+\tau\right) / \sigma}\right) \Theta_{1} \bar{\alpha}_{k} \\
& \quad+c_{k} d_{k}\left|u_{0}\right|^{q_{k}}\left|\xi_{k}\right|^{\left(4 l \sigma-\tau-r_{k}\right) / \sigma}\left|x_{k+1}\right|^{p_{k}} \leq \frac{1}{13} \sum_{i=1}^{k-1} \xi_{i}^{4 l} \\
& \quad+\sigma_{k 4} \Theta_{1} \xi_{k}^{4 l}+\bar{c}_{k} d_{k}\left|u_{0}\right|^{q_{k}}\left|\xi_{k}\right|^{\left(4 l \sigma-\tau-r_{k}\right) / \sigma} \\
& \quad \cdot\left|\xi_{k+1}\right|^{\left(r_{k}+\tau\right) / \sigma}+\bar{c}_{k} d_{k}\left|u_{0}\right|^{q_{k}}\left|\xi_{k}\right|^{\left(4 l \sigma-\tau-r_{k}\right) / \sigma} \\
& \quad \cdot\left|x_{k+1}^{*}\right|^{p_{k}},
\end{aligned}
$$

where $\sigma_{k 4} \geq 0$ is a smooth function. Based on (13), one has

$$
\begin{aligned}
& \left|\frac{\partial\left(\left\lceil\left. x_{k}^{*}\right|^{\sigma / r_{k}}\right)\right.}{\partial x_{i}}\right| \leq\left|\frac{\partial\left(\sum_{l=1}^{k-1} \prod_{j=l}^{k-1} \phi_{j}^{\sigma / r_{j+1}}\left\lceil\left. x_{l}\right|^{\sigma / r_{l}}\right)\right.}{\partial x_{i}}\right| \\
& \leq \prod_{j=i}^{k-1} \phi_{j}^{\sigma / r_{j+1}} \frac{\sigma}{r_{i}}\left(\left|\xi_{i}\right|^{\left(\sigma-r_{i}\right) / \sigma}+\phi_{i-1}^{\left(\sigma-r_{i}\right) / r_{i}}\left|\xi_{i-1}\right|^{\left(\sigma-r_{i}\right) / \sigma}\right) \\
& \quad+\sum_{l=1}^{k-1}\left|\frac{\partial\left(\prod_{j=i}^{k-1} \phi_{j}^{\sigma / r_{j+1}}\right)}{\partial x_{i}}\right|\left(\left|\xi_{l}\right|+\phi_{l-1}^{\sigma / r_{l}}\left|\xi_{l-1}\right|\right) .
\end{aligned}
$$


Similarly, by using (13), (A.4), (A.23), (A.26), Remark 14, and Lemma 4,

$$
\begin{aligned}
& \sum_{i=1}^{k-1} \frac{\partial W_{k}}{\partial x_{i}}\left(d_{i} u_{0}^{q_{i}} x_{i+1}^{p_{i}}+f_{i}\right) \\
& \leq \sum_{i=1}^{k-1} \sum_{j=1}^{i+1} \Theta_{1}\left(\left|\xi_{j}\right|^{\left(r_{i}+\tau\right) / \sigma}+\phi_{j-1}^{\left(r_{i}+\tau\right) / r_{i}}\left|\xi_{j-1}\right|^{\left(r_{i}+\tau\right) / \sigma}\right) \\
& \cdot F \times \frac{4 l \sigma-\tau-r_{k}}{\sigma} 2^{1-r_{k} / \sigma}\left|\frac{\partial\left(\left\lceil\left. x_{k}^{*}\right|^{\sigma / r_{k}}\right)\right.}{\partial x_{i}}\right| \\
& \cdot\left|\xi_{k}\right|^{((4 l-1) \sigma-\tau) / \sigma} \leq \frac{1}{13} \sum_{i=1}^{k-1} \xi_{i}^{4 l}+\sigma_{k 5} \Theta_{1} \xi_{k}^{4 l}
\end{aligned}
$$

where $\sigma_{k 5} \geq 0$ is a smooth function and $F \triangleq \max \left\{u_{0}^{q_{i}}(1-\right.$ $\left.\left.\bar{c}_{i}\right) \bar{\lambda}_{i, 2}, \bar{\alpha}_{i}\right\}$. Because of (A.11), (A.19), (A.24), Remark 14, and Lemma 4 , the following always holds:

$$
\begin{aligned}
& \frac{1}{2}\left|\frac{\partial^{2} W_{k}}{\partial x_{k} \partial x_{0}}\right|\left|g_{k}\right|\left|g_{0}^{\top}\right| \leq \sum_{i=1}^{k} \bar{\beta}_{0} \bar{\beta}_{k}\left|x_{0}\right| \Theta_{1} \\
& \quad \cdot \frac{4 l \sigma-\tau-r_{k}}{2 \sigma}\left|\xi_{k}\right|^{\left((4 l-1) \sigma-\tau-r_{k}\right) / \sigma}\left|\frac{\partial\left(\left\lceil x_{k}^{*}\right]^{\sigma / r_{k}}\right)}{\partial x_{0}}\right| \\
& \quad \cdot\left(\left|\xi_{i}\right|^{\left(2 r_{k}+\tau\right) / 2 \sigma}+\phi_{i-1}^{\left(2 r_{k}+\tau\right) / 2 r_{i}}\left|\xi_{i-1}\right|^{\left(2 r_{k}+\tau\right) / 2 \sigma}\right) \leq \frac{1}{13} \\
& \quad \cdot \sum_{i=1}^{k-1} \xi_{i}^{4 l}+\sigma_{k 6} \Theta_{1} \xi_{k}^{4 l},
\end{aligned}
$$

where $\sigma_{k 6} \geq 0$ is a smooth function. Using (A.7), (A.10), (A.24), (A.26), and Lemma 4, one has

$$
\begin{aligned}
& \frac{1}{2} \sum_{i=1}^{k-1}\left|\frac{\partial^{2} W_{k}}{\partial x_{k} \partial x_{i}}\right|\left|g_{k}\right|\left|g_{i}^{\top}\right| \leq \frac{1}{2} \\
& \quad \cdot \sum_{i=1}^{k-1} \sum_{l=1}^{k} \sum_{j=1}^{i} \bar{\beta}_{i} \bar{\beta}_{k} \Theta_{1} \frac{4 l \sigma-\tau-r_{k}}{2 \sigma}\left|\xi_{k}\right|^{\left((4 l-1) \sigma-\tau-r_{k}\right) / \sigma} \\
& \quad \times\left|\frac{\partial\left(\left\lceil\left. x_{k}^{*}\right|^{\sigma / r_{k}}\right)\right.}{\partial x_{i}}\right|\left(\left|\xi_{l}\right|^{\left(2 r_{k}+\tau\right) / 2 \sigma}\right. \\
& \left.\quad+\phi_{l-1}^{\left(2 r_{k}+\tau\right) / 2 r_{k}}\left|\xi_{l-1}\right|^{\left(2 r_{k}+\tau\right) / 2 \sigma}\right) \times\left(\left|\xi_{j}\right|^{\left(2 r_{i}+\tau\right) / 2 \sigma}\right. \\
& \left.\quad+\phi_{j-1}^{\left(2 r_{i}+\tau\right) / 2 r_{i}}\left|\xi_{j-1}\right|^{\left(2 r_{i}+\tau\right) / 2 \sigma}\right) \leq \frac{1}{13} \sum_{i=1}^{k-1} \xi_{i}^{4 l} \\
& \quad+\sigma_{k 7} \Theta_{1} \xi_{k}^{4 l}, \\
& \left.\frac{1}{2}\left|\frac{\partial^{2} W_{k}}{\partial x_{k}^{2} \mid}\right| g_{k}\right|^{2} \leq \sum_{i=1}^{k} \bar{\beta}_{k}^{2} \Theta_{1} \\
& \quad \frac{4 l \sigma+q_{k}-r_{k}}{2 \sigma}\left|\xi_{k}\right|^{\left((4 l-1) \sigma-\tau-r_{k}\right) / \sigma}\left|x_{k}\right|^{(\sigma+\tau) / r_{k}}
\end{aligned}
$$

$$
\begin{aligned}
& \times\left(\left|\xi_{i}\right|^{\left(2 r_{k}+\tau\right) / 2 \sigma}+\phi_{i-1}^{\left(2 r_{k}+\tau\right) / 2 r_{k}}\left|\xi_{i-1}\right|^{\left(2 r_{k}+\tau\right) / 2 \sigma}\right)^{2} \\
& \leq \frac{1}{13} \sum_{i=1}^{k-1} \xi_{i}^{4 l}+\sigma_{k 8} \Theta_{1} \xi_{k}^{4 l},
\end{aligned}
$$

where $\sigma_{k 7} \geq 0$ and $\sigma_{k 8} \geq 0$ are smooth functions. In terms of (13) and (A.19), it follows that

$$
\begin{aligned}
& \left|\frac{\partial^{2}\left(\left\lceil x_{k}^{*}\right\rceil^{\sigma / r_{k}}\right)}{\partial x_{i} \partial x_{0}}\right| \\
& \leq\left|\frac{\partial^{2} \phi_{k-1}^{\sigma / r_{k}}}{\partial x_{i} \partial x_{0}}\right|\left|\xi_{k-1}\right| \\
& +\sum_{j=1}^{k-2} \frac{\partial\left(\prod_{l=j+1}^{k-1}\left|\phi_{l}\right|^{\sigma / r_{l+1}}\left|\partial \phi_{j}^{\sigma / r_{j+1}} / \partial x_{0}\right|\right)}{\partial x_{i}}\left|\xi_{j}\right| \\
& +\sum_{j=1}^{k-2} \prod_{l=j+1}^{k-1}\left|\phi_{l}\right|^{\sigma / r_{l+1}}\left|\frac{\partial \phi_{j}^{\sigma / r_{j+1}}}{\partial x_{0}}\right|\left|\frac{\partial \xi_{j}}{\partial x_{i}}\right| \\
& +\left|\frac{\partial \xi_{k-1}}{\partial x_{i}}\right|\left|\frac{\partial \phi_{k-1}^{\sigma / r_{k}}}{\partial x_{0}}\right| \\
& \leq\left|\frac{\partial^{2} \phi_{k-1}^{\sigma / r_{k}}}{\partial x_{i} \partial x_{0}}\right|\left|\xi_{k-1}\right| \\
& +\sum_{j=1}^{k-2} \frac{\partial\left(\prod_{l=j+1}^{k-1}\left|\phi_{l}\right|^{\sigma / r_{l+1}}\left|\partial \phi_{j}^{\sigma / r_{j+1}} / \partial x_{0}\right|\right)}{\partial x_{i}}\left|\xi_{j}\right| \\
& +\sum_{j=1}^{k-2} \prod_{l=j+1}^{k-1}\left|\phi_{l}\right|^{\sigma / r_{l+1}}\left|\frac{\partial \phi_{j}^{\sigma / r_{j+1}}}{\partial x_{0}}\right|\left|\frac{\partial\left(\left\lceil\left. x_{j}^{*}\right|^{\sigma / r_{j}}\right)\right.}{\partial x_{i}}\right| \\
& +\left|\frac{\partial\left(\left\lceil x_{k-1}^{*}\right\rceil^{\sigma / r_{k-1}}\right)}{\partial x_{i}}\right|\left|\frac{\partial \phi_{k-1}^{\sigma / r_{k}}}{\partial x_{0}}\right| \text {. }
\end{aligned}
$$

Combining (A.12), (A.24), (A.26), (A.31), Remark 14, and Lemma 4 yields

$$
\begin{aligned}
& \frac{1}{2} \sum_{i=1}^{k-1}\left|\frac{\partial^{2} W_{k}}{\partial x_{i} \partial x_{0}}\right|\left|g_{i}\right|\left|g_{0}^{\top}\right| \leq \sum_{i=1}^{k-1} \sum_{j=1}^{i} 2^{-r_{k} / \sigma} \bar{\beta}_{0} \bar{\beta}_{i}\left|x_{0}\right| \Theta_{1} \\
& . \frac{4 l \sigma-\tau-r_{k}}{\sigma}\left|\xi_{k}\right|^{((4 l-2) \sigma-\tau) / \sigma} \\
& \cdot\left(\left|\xi_{j}\right|^{\left(2 r_{i}+\tau\right) / 2 \sigma}+\phi_{j-1}^{\left(2 r_{i}+\tau\right) / 2 \sigma}\left|\xi_{j-1}\right|^{\left(2 r_{i}+\tau\right) / 2 r_{i}}\right) \\
& \quad \times\left(\frac{(4 l-1) \sigma-\tau-r_{k}}{\sigma}\left|\frac{\partial\left(\left\lceil\left. x_{k}^{*}\right|^{\sigma / r_{k}}\right)\right.}{\partial x_{i}}\right| \sum_{l=1}^{k-1} \psi_{l, 1}\left|\xi_{j}\right|\right.
\end{aligned}
$$




$$
\left.+\left|\frac{\partial^{2}\left(\left\lceil x_{k}^{*}\right\rceil^{\sigma / r_{k}}\right)}{\partial x_{i} \partial x_{0}}\right|\left|\xi_{k}\right|\right) \leq \frac{1}{13} \sum_{i=1}^{k-1} \xi_{i}^{4 l}+\sigma_{k 9} \Theta_{1} \xi_{k}^{4 l}
$$

where $\sigma_{k 9} \geq 0$ is a smooth function. Exactly following the same procedure, tedious calculations conclude that

$$
\begin{aligned}
& \frac{1}{2} \sum_{i=1}^{k-1} \sum_{j=1}^{i}\left|\frac{\partial^{2} W_{k}}{\partial x_{i}^{2}}\right|\left|g_{i}\right|^{2} \\
& \leq \sum_{i=1}^{k-1} \sum_{j=1}^{i} \bar{\beta}_{i}^{2} \Theta_{1} 2^{-r_{k} / \sigma}\left|\xi_{k}\right|^{((4 l-2) \sigma-\tau) / \sigma} \\
& \frac{4 l \sigma-\tau-r_{k}}{\sigma}\left(\left(\frac{\partial\left(\left\lceil x_{k}^{*}\right\rceil^{\sigma / r_{k}}\right)}{\partial x_{i}}\right)^{2}\right. \\
& \left.\times \frac{(4 l-1) \sigma-\tau-r_{k}}{\sigma}+\left|\frac{\partial^{2}\left(\left\lceil x_{k}^{*}\right\rceil^{\sigma / r_{k}}\right)}{\partial x_{i}^{2}}\right|\left|\xi_{k}\right|\right) \\
& \times\left(\left|\xi_{j}\right|^{\left(2 r_{i}+\tau\right) / 2 \sigma}+\phi_{j-1}^{\left(2 r_{i}+\tau\right) / 2 r_{i}}\left|\xi_{j-1}\right|^{\left(2 r_{i}+\tau\right) / 2 \sigma}\right)^{2} \\
& \leq \frac{1}{13} \sum_{i=1}^{k-1} \xi_{i}^{4 l}+\sigma_{k 10} \Theta_{1} \xi_{k}^{4 l} \\
& \sum_{i, j=1, i \neq j}^{k-1} \frac{1}{2}\left|\frac{\partial^{2} W_{k}}{\partial x_{i} \partial x_{j}}\right|\left|g_{i}\right|\left|g_{j}^{\top}\right| \leq \sum_{i, j=1, i \neq j}^{k-1} \sum_{l=1}^{i} \sum_{h=1}^{j} 2^{-r_{k} / \sigma} \\
& \frac{4 l \sigma-\tau-r_{k}}{\sigma} \bar{\beta}_{i} \bar{\beta}_{j} \Theta_{1}\left|\xi_{k}\right|^{((4 l-2) \sigma-\tau) / \sigma} \\
& \times\left(\frac{(4 l-1) \sigma-\tau-r_{k}}{\sigma}\left|\frac{\partial\left(\left\lceil x_{k}^{*}\right\rceil^{\sigma / r_{k}}\right)}{\partial x_{i}}\right|\right. \\
& \left.\cdot\left|\frac{\partial\left(\left\lceil x_{k}^{*}\right\rceil^{\sigma / r_{k}}\right)}{\partial x_{j}}\right|+\left|\frac{\partial^{2}\left(\left\lceil x_{k}^{*}\right\rceil^{\sigma / r_{k}}\right)}{\partial x_{i} \partial x_{j}}\right|\left|\xi_{k}\right|\right) \\
& \cdot\left(\left|\xi_{l}\right|^{\left(2 r_{i}+\tau\right) / 2 \sigma}+\phi_{l-1}^{\left(2 r_{i}+\tau\right) / 2 r_{i}}\left|\xi_{l-1}\right|^{\left(2 r_{i}+\tau\right) / 2 \sigma}\right) \\
& \times\left(\left|\xi_{h}\right|^{\left(2 r_{j}+\tau\right) / 2 \sigma}+\phi_{h-1}^{\left(2 r_{j}+\tau\right) / 2 r_{j}}\left|\xi_{h-1}\right|^{\left(2 r_{j}+\tau\right) / 2 \sigma}\right) \\
& \leq \frac{1}{13} \sum_{i=1}^{k-1} \xi_{i}^{4 l}+\sigma_{k 11} \Theta_{1} \xi_{k}^{4 l}
\end{aligned}
$$

where $\sigma_{k 10} \geq 0$ and $\sigma_{k 11} \geq 0$ are smooth functions. At last, by using Lemma 4,

$$
\begin{gathered}
\bar{c}_{k-1} d_{k-1}\left|u_{0}\right|^{q_{k-1}}\left|\xi_{k-1}\right|^{\left(4 l \sigma-\tau-r_{k-1}\right) / \sigma}\left|\xi_{k}\right|^{\left(r_{k-1}+\tau\right) / \sigma} \\
\leq \frac{1}{13} \xi_{k-1}^{4 l}+\sigma_{k 12} \Theta_{1} \xi_{k}^{4 l}
\end{gathered}
$$

where $\sigma_{k 12}$ is a position constant. Define the nonnegative smooth function $\sigma_{k}=\sum_{i=1}^{12} \sigma_{k i}$, and put (A.18), (A.20), (A.22), (A.25), (A.27)-(A.30), and (A.32)-(A.35) together, the proof of Proposition 18 is completed.

Proof of Proposition 19. By (31), Proposition 17, and Lemma 2, Proposition 19 is proved.

\section{Data Availability}

The data supporting the conclusions of our article are some open access articles that have been properly cited, and the readers can easily obtain these articles to verify the conclusions, replicate the analysis, and conduct secondary analyses. Therefore, we do not create a publicly available data repository.

\section{Conflicts of Interest}

The authors declare that they have no conflicts of interest.

\section{Acknowledgments}

This work was supported by Taishan Scholar Project of Shandong Province of China (no. ts201712040), National Natural Science Foundation of China (no. 61673242), and Shandong Provincial Natural Science Foundation of China (no. ZR2016FM10).

\section{References}

[1] M. Krstic and H. Deng, Stabilization of Nonlinear Uncertain Systems, Springer-Verlag, New York, NY, USA, 1998.

[2] X. Mao, Stochastic Differential Equations and Applications, Horwood Publishing, Chichester, UK, 2007.

[3] Z. Pan and T. Basar, "Backstepping controller design for nonlinear stochastic systems under a risk-sensitive cost criterion," SIAM Journal on Control and Optimization, vol. 37, no. 3, pp. 957-995, 1999.

[4] Y.-G. Liu and J.-F. Zhang, "Practical output-feedback risksensitive control for stochastic nonlinear systems with stable zero-dynamics," SIAM Journal on Control and Optimization, vol. 45, no. 3, pp. 885-926, 2006.

[5] S.-J. Liu, J.-F. Zhang, and Z.-P. Jiang, "Decentralized adaptive output-feedback stabilization for large-scale stochastic nonlinear systems," Automatica, vol. 43, no. 2, pp. 238-251, 2007.

[6] S.-J. Liu, Z.-P. Jiang, and J.-F. Zhang, "Global output-feedback stabilization for a class of stochastic non-minimum-phase nonlinear systems," Automatica, vol. 44, no. 8, pp. 1944-1957, 2008.

[7] L. Liu and X.-J. Xie, "State feedback stabilization for stochastic feedforward nonlinear systems with time-varying delay," Automatica, vol. 49, no. 4, pp. 936-942, 2013.

[8] Q. Wang and C. Wei, "Decentralized robust adaptive output feedback control of stochastic nonlinear interconnected systems with dynamic interactions," Automatica, vol. 54, pp. 124134,2015

[9] K. Zhang, C.-R. Zhao, and X.-J. Xie, "Global output feedback stabilisation of stochastic high-order feedforward nonlinear systems with time-delay," International Journal of Control, vol. 88, no. 12, pp. 2477-2487, 2015. 
[10] C.-R. Zhao, K. Zhang, and X.-J. Xie, "Output feedback stabilization of stochastic feedforward nonlinear systems with input and state delay," International Journal of Robust and Nonlinear Control, vol. 26, no. 7, pp. 1422-1436, 2016.

[11] M.-M. Jiang, K.-M. Zhang, and X.-J. Xie, "Output feedback stabilisation of stochastic nonlinear time-delay systems with unknown output function," International Journal of Systems Science, vol. 48, no. 11, pp. 2262-2271, 2017.

[12] H. Sun, Y. Li, G. Zong, and L. Hou, "Disturbance attenuation and rejection for stochastic Markovian jump system with partially known transition probabilities," Automatica, vol. 89, pp. 349-357, 2018.

[13] A. Ferrara, L. Giacomini, and C. Vecchio, "Control of nonholonomic systems with uncertainties via second-order sliding modes," International Journal of Robust and Nonlinear Control, vol. 18, no. 4-5, pp. 515-528, 2008.

[14] F. Z. Gao, F. S. Yuan, H. J. Yao, and X. W. Mu, "Adaptive stabilization of high order nonholonomic systems with strong nonlinear drifts," Applied Mathematical Modelling, vol. 35, no. 9, pp. 4222-4233, 2011.

[15] F. Z. Gao, F. S. Yuan, and H. J. Yao, "Robust adaptive control for nonholonomic systems with nonlinear parameterization," Nonlinear Analysis: Real World Applications, vol. 11, no. 4, pp. 3242-3250, 2010.

[16] W. Lin, R. Pongvuthithum, and C. Qian, "Control of highorder nonholonomic systems in power chained form using discontinuous feedback," Institute of Electrical and Electronics Engineers Transactions on Automatic Control, vol. 47, no. 1, pp. 108-115, 2002.

[17] Q. H. Du, C. L. Wang, and G. Wang, "Adaptive state-feedback stabilization of stochastic high-order nonholonomic systems with nonlinear parameterization," Transactions of the Institute of Measurement and Control, vol. 37, no. 4, pp. 536-549, 2015.

[18] W. Lin and C. Qian, "Adaptive control of nonlinearly parameterized systems: a nonsmooth feedback framework," IEEE Transactions on Automatic Control, vol. 47, no. 6, pp. 757-774, 2002.

[19] B. Yang and W. Lin, "Robust output feedback stabilization of uncertain nonlinear systems with uncontrollable and unobservable linearization," IEEE Transactions on Automatic Control, vol. 50, no. 5, pp. 619-630, 2005.

[20] F. Gao and Y. Wu, "Global stabilisation for a class of more general high-order time-delay nonlinear systems by output feedback," International Journal of Control, vol. 88, no. 8, pp. 1540-1553, 2015.

[21] D. Zhang, C. Wang, G. Wei, and H. Chen, "Output feedback stabilization for stochastic nonholonomic systems with nonlinear drifts and Markovian switching," Asian Journal of Control, vol. 16, no. 6, pp. 1679-1692, 2014.

[22] F. Gao and F. Yuan, "Adaptive stabilization of stochastic nonholonomic systems with nonlinear parameterization," Applied Mathematics and Computation, vol. 219, no. 16, pp. 8676-8686, 2013.

[23] F. Gao, F. Yuan, and Y. Wu, "Adaptive stabilization for a class of stochastic nonlinearly parameterized nonholonomic systems with unknown control coefficients," Asian Journal of Control, vol. 16, no. 6, pp. 1829-1838, 2014.

[24] F. Gao and F. Yuan, "Finite-time stabilization of stochastic nonholonomic systems and its application to mobile robot," Abstract and Applied Analysis, vol. 2012, Article ID 361269, 18 pages, 2012.
[25] F. Gao, F. Yuan, J. Zhang, and Y. Wu, "Further result on finite-time stabilization of stochastic nonholonomic systems," Abstract and Applied Analysis, vol. 2013, 8 pages, 2013.

[26] S. S. Ge, Z. P. Wang, and T. H. Lee, "Adaptive stabilization of uncertain nonholonomic systems by state and output feedback," Automatica, vol. 39, no. 8, pp. 1451-1460, 2003.

[27] H. Deng, M. Krstić, and R. J. Williams, "Stabilization of stochastic nonlinear systems driven by noise of unknown covariance," IEEE Transactions on Automatic Control, vol. 46, no. 8, pp. 12371253, 2001.

[28] M.-Y. Cui, X.-J. Xie, and Z.-J. Wu, "Dynamics modeling and tracking control of robot manipulators in random vibration environment," Institute of Electrical and Electronics Engineers Transactions on Automatic Control, vol. 58, no. 6, pp. 1540-1545, 2013.

[29] C. Zhao and X. Xie, "Global stabilization of stochastic highorder feedforward nonlinear systems with time-varying delay," Automatica, vol. 50, no. 1, pp. 203-210, 2014.

[30] X. Xie, N. Duan, and C. Zhao, "A combined homogeneous domination and sign function approach to output-feedback stabilization of stochastic high-order nonlinear systems," IEEE Transactions on Automatic Control, vol. 59, no. 5, pp. 1303-1309, 2014.

[31] H. Ito and Y. Nishimura, "Stability of stochastic nonlinear systems in cascade with not necessarily unbounded decay rates," Automatica, vol. 62, no. 12, pp. 51-64, 2015.

[32] X.-J. Xie, X.-H. Zhang, and K. Zhang, "Finite-time state feedback stabilisation of stochastic high-order nonlinear feedforward systems," International Journal of Control, vol. 89, no. 7, pp. 1332-1341, 2016.

[33] H. Ito and Y. Nishimura, "An iISS framework for stochastic robustness of interconnected nonlinear systems," Institute of Electrical and Electronics Engineers Transactions on Automatic Control, vol. 61, no. 6, pp. 1508-1523, 2016.

[34] X. Xie and M. Jiang, "Output feedback stabilization of stochastic feedforward nonlinear time-delay systems with unknown output function," International Journal of Robust and Nonlinear Control, vol. 28, no. 1, pp. 266-280, 2018.

[35] M.-M. Jiang and X.-J. Xie, "Finite-time stabilization of stochastic low-order nonlinear systems with FT-SISS inverse dynamics," International Journal of Robust and Nonlinear Control, vol. 28, no. 6, pp. 1960-1972, 2018. 


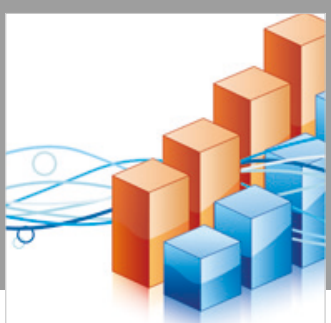

Advances in

Operations Research

\section{-n-m}
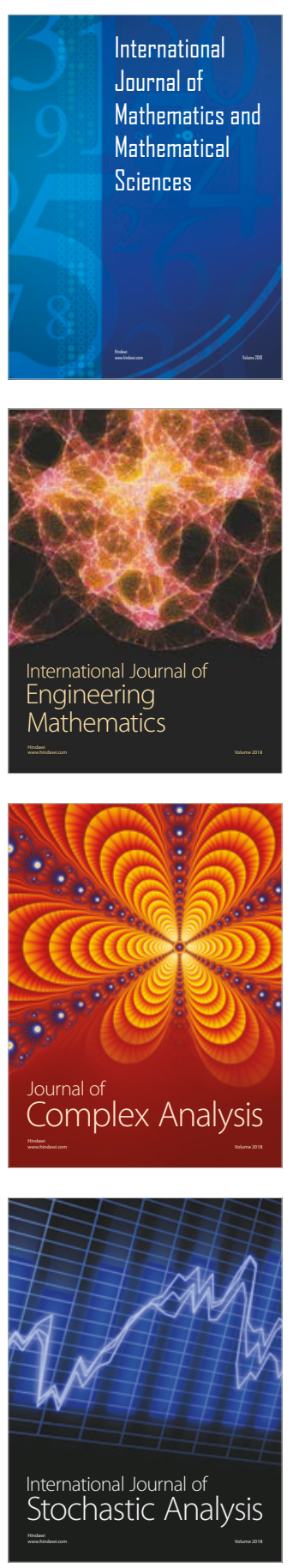
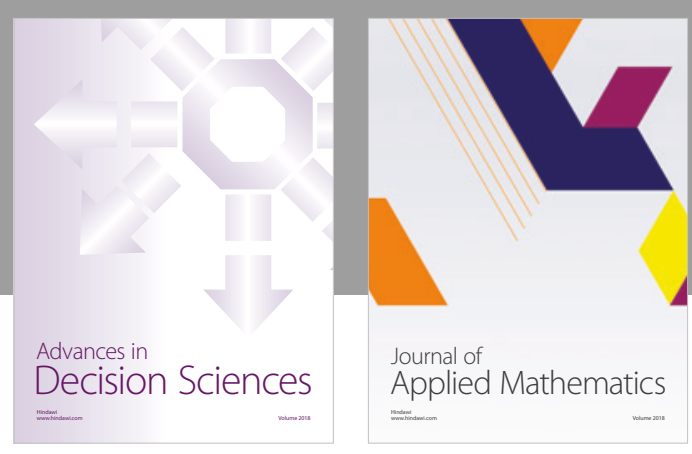

Journal of

Applied Mathematics
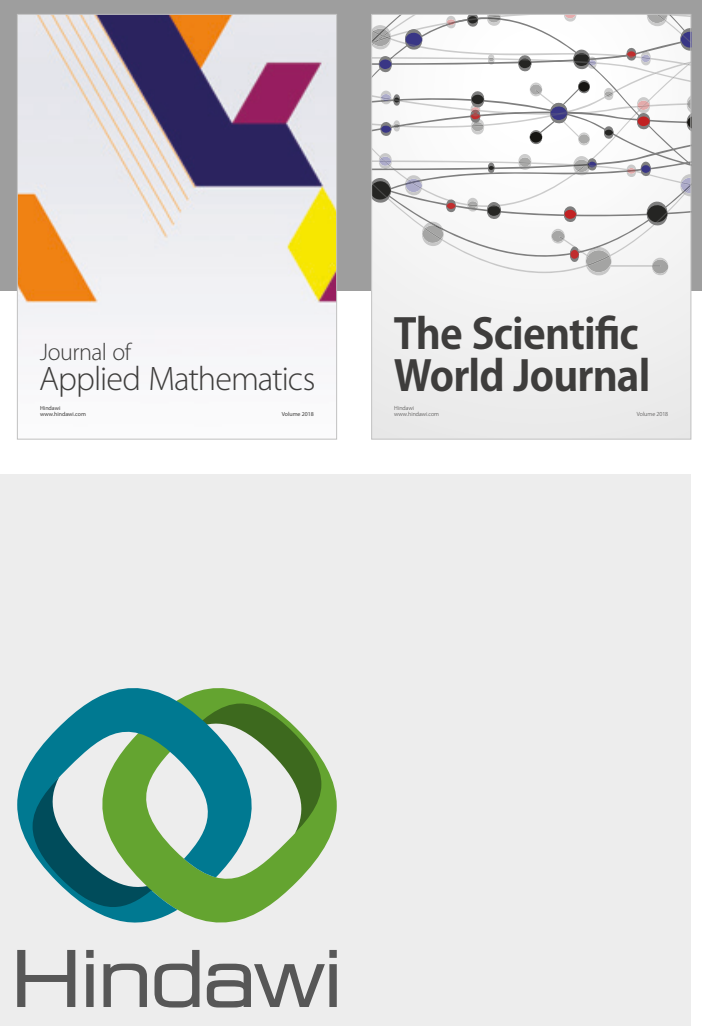

Submit your manuscripts at

www.hindawi.com

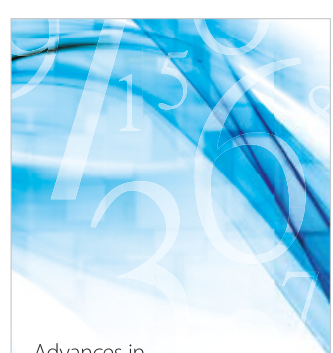

Advances in
Numerical Analysis
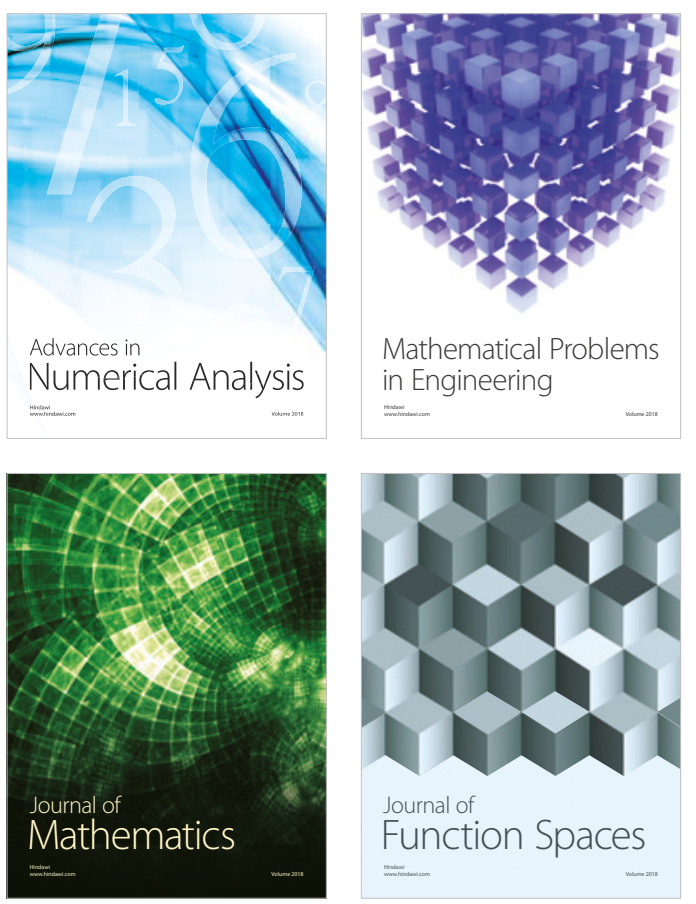

Mathematical Problems in Engineering

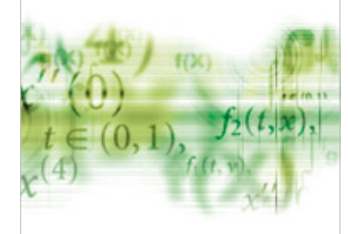

International Journal of

Differential Equations

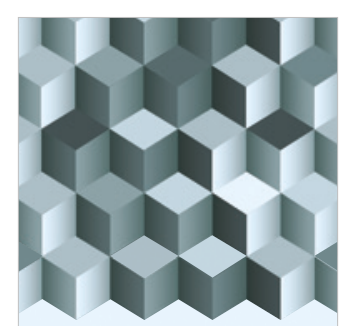

Journal of

Function Spaces

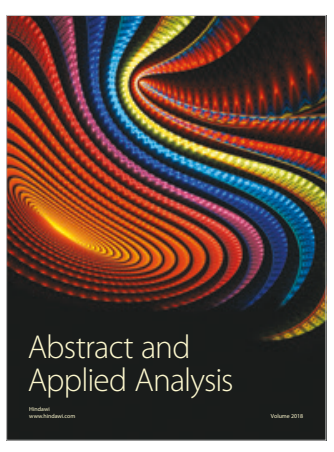

The Scientific

World Journal

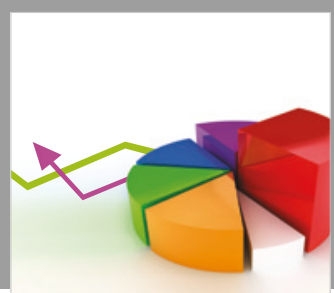

Journal of

Probability and Statistics
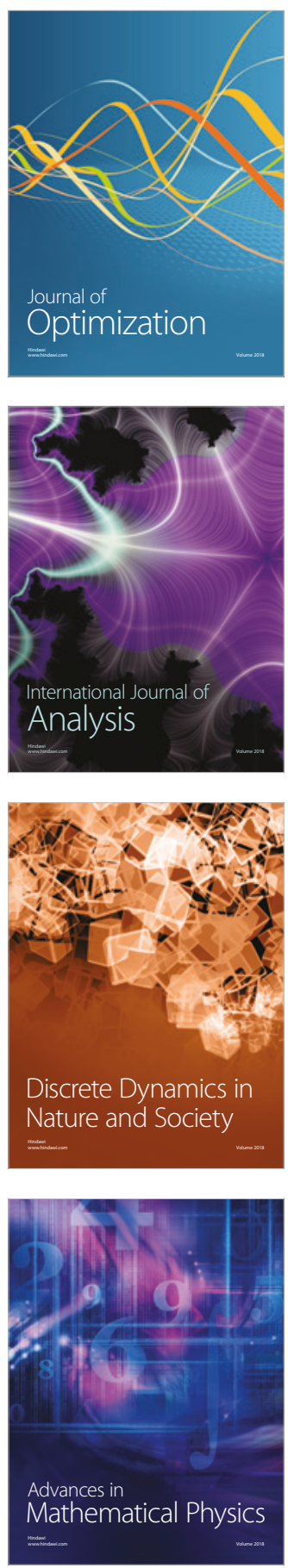